\title{
Development of the First Tractable Genetic System for Parvimonas micra, a Ubiquitous Pathobiont in Human Dysbiotic Disease
}

Dustin L. Higashi ${ }^{1}$, Sean McGuire ${ }^{2}$, Yasser Abdelrahman ${ }^{1}$, Zhengzhong Zou , Hua

Qin ${ }^{1}$, David Anderson', Elizabeth A. Palmer ${ }^{2}$, Jens Kreth ${ }^{1,3}$, and Justin Merritt

5 'Department of Restorative Dentistry, Oregon Health and Science University, Portland, OR USA 97239

2

Department of Pediatric Dentistry, Oregon Health and Science University, Portland, OR USA 97201

${ }^{3}$ Department of Molecular Microbiology and Immunology, Oregon Health and

10 Science University 97239

*Corresponding Author: Email: merrittj@ohsu.edu

Running Title: A Genetic System for Parvimonas micra

35 Keywords: Gram-positive bacteria, Parvimonas micra, genetics, natural competence 


\section{Abstract}

Parvimonas micra is a Gram-positive obligate anaerobe and a typical member of the human microbiome. P. micra is among the most highly enriched species at numerous sites of mucosal dysbiotic disease and is closely associated with the

45 development of multiple types of malignant tumors. Despite its strong association with disease, surprisingly little is known about $P$. micra pathobiology, which is directly attributable to its longstanding genetic intractability. To address this problem, we directly isolated a collection of $P$. micra strains from odontogenic abscess clinical specimens and then screened these isolates for natural

50 competence. Amazingly, all of the $P$. micra clinical isolates exhibited various levels of natural competence, including the reference strain ATCC 33270. By exploiting this ability, we were able to employ cloning-independent methodologies to engineer and complement a variety of targeted chromosomal genetic mutations directly within low passage clinical isolates. To create the first $P$. micra genetic 55 system, we employed renilla-based bioluminescence for highly sensitive reporter studies. This reporter system was then applied for the development of the novel Theo + theophylline-inducible riboswitch for tunable gene expression studies over a broad dynamic range. Finally, we demonstrate the feasibility of generating Mariner-based Tn-seq libraries for forward genetic screening in P. micra. With the

60 availability of a highly efficient transformation protocol and the current suite of genetic tools, $P$. micra should now be considered as a fully genetically tractable organism suitable for molecular genetic research. The methods presented here provide a clear path to investigate the understudied role of $P$. micra in polymicrobial infections and tumorigenesis. 


\section{Introduction}

70 Parvimonas micra is a Gram-positive obligate anaerobe from the largely uncharacterized Tissierellia class of the Firmicutes phylum. Initially grouped within the Peptostreptococcus genus, a taxonomic reclassification revealed its distinction within the Firmicutes $[1,2]$. P. micra is a common commensal of microbiomes at various mucosal sites in the body, including the oral cavity,

75 gastrointestinal tract, respiratory system, and female urogenital tract. P. micra is a surprisingly common and abundant species detected in numerous epidemiological studies of different mucosal inflammatory diseases [3]. In addition, P. micra is among the most common sources of Gram-positive anaerobic cocci (GPAC) sepsis. It is a major constituent of numerous types of systemic abscesses, and it

80 exhibits a strong association with a variety of malignant tumors [4-7]. Indeed, its presence has even been proposed as a discriminating biomarker for colorectal cancer, gastric cancer, and oral cancer $[4,8,9]$. In the oral cavity, P. micra is highly enriched in periodontitis lesions, infected root canals, and is especially prevalent in polymicrobial odontogenic abscesses [10-15].

The mechanisms by which $P$. micra contributes to human health and disease remain largely enigmatic, as there is a severe paucity of literature describing its molecular genetics [7]. Furthermore, this organism has been historically challenging to identify in clinical microbiology laboratories, largely due to its

90 fastidious nature and slow growth rate [3]. Reports suggest that $P$. micra frequently serves as a major pathobiont involved in pathogenic synergism with other members of the microbiome. A recent in vitro study illustrated how P. micra can augment the growth of Porphyromonas gingivalis and enhance its production of secreted proteolytic gingipains [16]. In vivo studies demonstrated an enhanced

95 transmissibility of pus generated from P. micra/Prevotella co-infections compared to their respective mono-infections [17]. P. micra can also coaggregate with the oral pathobionts Treponema denticola and Fusobacterium nucleatum, implicating a potential role in polymicrobial oral biofilm formation $[18,19]$.

100 Despite its strong association with a broad diversity of mucosal inflammatory diseases, $P$. micra remains vastly understudied, largely due to its genetic intractability. Only a single report of targeted mutagenesis has ever been described for this organism and this was accomplished via electroporation of a suicide vector [20]. The lack of follow-up genetic studies underscores the significant challenges 105 associated with genetically manipulating $P$. micra. Such problems have remained a significant deterrent for detailed mechanistic studies of $P$. micra pathobiology. To address this problem, we present the first efficacious $P$. micra genetic system, which is founded upon a newly discovered natural competence ability in this organism. By exploiting this ability, we were able to apply cloning-independent 
110 methodologies to engineer a variety of targeted chromosomal genetic modifications using low passage clinical isolates. The efficacy of our P. micra transformation protocol further supported the development of the first tractable genetic system for this species. With the genetic toolbox presented here, a complete molecular genetic system is in place to reliably interrogate P. micra

115 pathobiology.

\section{Results}

120 Identification of Natural Competence in $\boldsymbol{P}$. micra clinical isolates

Recently, Liu and Hou described the only known report of targeted mutagenesis in P. micra [20]. This study employed a cloning-based methodology to create a mutagenesis construct introduced via electrotransformation of a suicide plasmid.

125 Due to the difficulties associated with P. micra electroporation, we were interested to determine whether a more reliable transformation approach could be developed using natural competence. To test this, we first isolated a collection of low passage clinical strains directly from odontogenic abscess specimens (Figure 1A). Following tooth extraction, abscess pus samples were collected into pre-reduced

130 transport media [21] and then spread onto PMM selective/differential agar media [12]. Candidate P. micra colonies were selected based upon the accumulation of black precipitate, then examined morphologically by microscopy, and finally confirmed with 16S rRNA sequencing (Figure 1A).

135 We next generated a selectable marker for use in DNA transformation assays by isolating a spontaneous rifampicin resistant mutant of the wild-type reference strain ATCC 33270 (Figure 1B). A resulting rifampicin resistant ( (if $^{\mathrm{R}}$ ) colony of ATCC 33270 was verified by sequence analysis of the rpoB gene encoding the beta subunit of RNA polymerase. A point mutation in this gene confirmed a

140 D501V substitution in RpoB (Figure 1C), which coincides with rifampicin resistance mutations detected in other bacterial species [22, 23]. Next, genomic DNA (gDNA) was extracted from both the wild-type strain ATCC 33270 (rif $^{\mathrm{S}}$ ) and the rifampicin resistant mutant of ATCC $33270\left(\right.$ rif $\left.^{R}\right)$ (Figure 1B) and then transformed into our collection of wild-type $P$. micra clinical isolates using a

145 transformation protocol similar to that previously employed for Veillonella parvula [24]. With this approach, we observed natural competence from the majority of strains tested, with particularly high transformation efficiencies in a number of isolates (Table 1). These results confirmed that $P$. micra is capable of developing natural competence. 


\section{Cloning-Independent Targeted Mutagenesis}

Following our discovery of $P$. micra natural competence, it was of interest to determine whether this ability could be exploited for engineering targeted

155 mutations in P. micra. For this, we chose the ermB erythromycin resistance cassette as a selectable marker [25]. An allelic replacement mutagenesis construct was created by Gibson assembly of PCR amplicons containing ermB flanked by one kb homologous fragments of the EF-Tu-encoding gene tuf and its downstream locus (Figure 2A, top). Transformation of this construct into the clinical strain A28

160 resulted in the insertion of ermB immediately downstream of the tuf open reading frame (ORF) through a double crossover allelic exchange event (Figures $2 \mathrm{~A}$ and B). Next, replicate constructs were generated for each isolate and then transformed into their respective strains, including the reference strain ATCC 33270. In each of these reactions, background erythromycin resistance was undetectable, whereas 4

165 out of 5 isolates, including ATCC 33270 exhibited detectable levels of natural competence (Figure 2C).

\section{Comparison of construct homology vs. transformation efficiency}

In an attempt to further bolster the transformation efficiencies of our isolates, we next tested a variety of mutagenesis constructs (Figure 3A) to examine the correlation between the sizes of the homologous DNA fragments and the resulting transformation efficiencies [26, 27]. Here, we focused on isolate A28, which

175 displayed the highest level of natural competence using $1 \mathrm{~kb}$ homologous fragments (Figure 2C). No transformants were detected in the negative control samples or when using mutagenesis constructs containing homologous flanking regions of $250 \mathrm{bp}$ (Figure 3B). An increase in the size of the homologous fragments from $1000 \mathrm{bp}$ to $1750 \mathrm{bp}$ resulted in $>100$-fold increase in

180 transformation efficiency. A further increase in homologous fragment length from $1750 \mathrm{bp}$ to $2500 \mathrm{bp}$ only resulted in an additional $\sim 15 \%$ increase in transformation efficiency, which suggested that transformation rates were approaching their maximum. Given the significant impact of larger homologous flanking regions upon the transformation rates of A28, we subsequently reexamined the

185 transformation-negative strain A1 (Figure 2C) using $2.5 \mathrm{~kb}$ homologous flanking arms. Using this larger construct, it was then possible to detect reliable, albeit low levels of transformation in this strain (Figure 3B). These results demonstrate that the size of construct homology plays a significant role for natural transformation efficiency in $P$. micra, with homologous arms of $\geq 1750 \mathrm{bp}$ being optimal for maximal rates of transformation.

\section{Targeted mutations of rec $A$ in $P$. micra}


195 We were next interested to determine whether our P. micra transformation protocol could be used for targeted allelic replacement mutagenesis of $\operatorname{rec} A$. RecA is a nonessential DNA-dependent ATPase that plays a central role in homologous DNA recombination and repair [28, 29]. Given the requirement for homologous recombination during allelic replacement mutagenesis, it was anticipated that a

200 rec $A$ mutant would exhibit a natural transformation defective phenotype, preventing subsequent mutagenesis using a marked linear DNA construct. Similar to other bacteria, we also anticipated a $\operatorname{rec} A$ mutant to exhibit highly increased sensitivity to DNA damage. We tested this in strain A28 by first transforming a rec $A$ deletion construct comprised of an erythromycin resistance cassette $(\mathrm{erm} B)$

205 flanked by one kb DNA fragments homologous to the upstream and downstream regions surrounding $r e c A$ (Figure $4 \mathrm{~A}$ top). This resulted in a double crossover allelic exchange deletion mutant in which $r e c A$ was replaced with ermB (Figure 4A middle and bottom). Since the transformation defects resulting from a rec $A$ mutation would likely preclude subsequent genetic complementation, it was

210 necessary to first create an ectopically expressed $r e c A$ knock-in mutant to serve as a recipient strain for a deletion of the native $\operatorname{rec} A$ gene. A mutagenesis construct comprised of the recA ORF followed by a kanamycin resistance cassette (aphAIII) [25] was ligated to $2.5 \mathrm{~kb}$ segments of the tuf gene and its downstream sequence (Figure 4B top). After transforming this construct into strain A28, a double

215 crossover event resulted in the insertion of an ectopic copy of the rec $A$ ORF and aphAIII directly downstream of tuf (Figure 4B middle and bottom). In this double rec $A$-expressing background, we subsequently deleted the native $r e c A$ gene using the same rec $A$ deletion construct described above (Figure 4A). Consequently, the resulting erythromycin/kanamycin resistant transformant harbored a deletion of

220 the endogenous $\operatorname{rec} A$, while also expressing an ectopic copy of $\operatorname{rec} A$ together with the tuf gene as part of an artificial operon. We subsequently compared the transformation efficiencies of these rec $A$ mutant strains using a PCR-generated amplicon of the rif $^{\mathrm{R}}$ rpoB gene previously generated in the strain ATCC 33270 (Figure 1B). The double recA-expressing strain (+A) and the complemented rec $A$ 225 deletion strain $(+\mathrm{A} / \Delta \mathrm{A})$ both exhibited similar transformation efficiencies to the wild-type (WT), whereas the $\Delta r e c A$ deletion strain $(\Delta \mathrm{A})$ yielded no detectable levels of natural competence (Figure 4C). As an independent verification of the $\operatorname{rec} A$ deletion phenotype, we further tested these strains for sensitivity to the genotoxic agent mitomycin $\mathrm{C}(\mathrm{MMC})$. As expected, the $\Delta r e c A$ strain $(\Delta \mathrm{A})$

230 displayed a greater sensitivity to $\mathrm{MMC}$ at all of the tested dosages $\left(0.5-4 \mu \mathrm{g} \mathrm{mL}^{-1}\right)$, with a $>3-\log$ reduced survival rate observed at the highest MMC dosage (Figure 4D). In agreement with the transformation results, both the single and double rec $A$ strains behaved similarly to the wild-type. Taken together, these results demonstrate the successful implementation of a highly efficient $P$. micra

235 mutagenesis protocol that can be employed for both targeted gene deletion and genetic complementation using naturally transformed PCR products. 


\section{Creation of a renilla luciferase reporter}

The high quantum yield and low background of luciferase-based reporter assays can provide a rapid and precise measure of gene expression over an exceptionally wide dynamic range. The superior signal-to-noise ratio of bioluminescent reporters has also supported noninvasive biophotonic imaging studies of mice orally

245 infected with human oral streptococci [30]. To determine whether similar luciferase reporters would also function in P. micra, we developed a constitutive green renilla luciferase expression strain by inserting an independently translated green renilla (ren $G$ ) luciferase gene and a kanamycin resistance cassette (aphAIII) immediately downstream of the $t u f$ gene (Figure 5A). Green renilla luciferase

250 (RenG) has been successfully employed in several species of oral Streptococcus and displays a much higher signal intensity compared to that of firefly luciferase [30]. Luciferase assays of this strain demonstrated extremely high levels of reporter activity that were $>5$-logs above the background readings of the unmodified parent strain A28 (Figure 5B). To gauge the detection limits and

255 precision of the luciferase reporter readings in this strain, we next examined the correlation between population size and luciferase bioluminescence values. A dilution series of the reporter exhibited an extremely strong linear correlation with bioluminescence, with precise reporter values detectable in as few as $10^{2}-10^{3}$ total CFU (Figure 5C). This suggests that luciferase reporter assays can be accurately

260 performed within an exceptionally wide range of $P$. micra cell densities.

\section{Tunable-induction system in P.micra.}

265 The efficacy of the renilla luciferase reporter subsequently provided an avenue for developing an inducible expression system in P. micra. Regulated gene expression systems are powerful tools for genetic and functional studies. Optimal systems display a low basal expression along with strong and tunable levels of induction. We first tested the xylose-inducible expression cassette Xyl-S1 developed for oral

270 Streptococcus species [31]. However, this only yielded nominal xylose-dependent regulation in $P$. micra. Therefore, we were curious whether a posttranscriptional regulatory system, such as those of ligand-inducible riboswitches [32], might perform better. To test this, we inserted the theophylline riboswitch [32] between the $\operatorname{ren} G$ ORF and an ectopic copy of the constitutively-expressed rpoB promoter.

275 This construct was then integrated onto the chromosome immediately downstream of the tuf gene transcription terminator to prevent transcriptional read-through from tuf (Fig. 6A). This strain was assayed for luciferase reporter activity over a range of theophylline concentrations, and we observed a titratable dose response curve with $\sim 9$-fold dynamic range (Figure 6C). This result is highly consistent 
280 with previous studies employing this riboswitch in other species [32]. Encouraged by the inducibility of this riboswitch in P. micra, we sought to improve upon its dynamic range by introducing a series of point mutations that were predicted to introduce a Rho-independent terminator-like element without triggering major changes to the overall riboswitch secondary structure (Figure 6B). Intriguingly,

285 this modified reporter strain displayed a greatly enhanced response to theophylline, resulting in a $>70$-fold dynamic range (Figure $6 \mathrm{C}$ ). Together, these results demonstrate the utility of $\operatorname{ren} G$-encoded luciferase as a sensitive reporter gene in $P$. micra as well as a riboswitch-based tunable gene expression system that exhibits low basal expression and robust inducibility.

\section{Development of in vitro transposon mutagenesis for Tn-seq studies}

Transposon sequencing (Tn-seq) is a powerful forward genetic screening tool that

295 has been successfully employed for genetic fitness studies of a number of pathogens [33] and pathobiont microbiome species [25, 34]. Given the high level of natural competence observed in P. micra, we were curious to determine the feasibility of implementing in vitro transposon mutagenesis using recombinant MarC9 transposase and the mini-Mariner transposon commonly used in Tn-seq

300 studies (Figure 7A-B) [25, 33, 35]. Following in vitro transposon mutagenesis of $P$. micra gDNA, the reactions were transformed directly into the wild-type strain A28. With this approach, we readily obtained $>6,000$ transposon mutants $/ \mu \mathrm{g}$ gDNA (Figure 7C and D). Considering that the $P$. micra genome is $<2 \mathrm{Mb}$, a single in vitro mutagenesis reaction with several $\mu \mathrm{g}$ of $P$. micra gDNA would be 305 sufficient to create densely saturated libraries of transposon insertions that are directly compatible with Tn-seq protocols. Overall, P. micra should be highly amenable to Tn-seq studies of its pathobiology. 


\section{Discussion}

$310 P$. micra is a notable pathobiont in the oral cavity due to its strong association with a variety of inflammatory oral diseases. $P$. micra is also commonly identified as a major constituent of acute and chronic infections at numerous other sites in the human body, including the abdomen, chest, spine, brain, blood, skin, and urogenital tract [36, 37]. Many P. micra infections have been described as

315 polymicrobial, though a number of reports have implicated it as the sole source of infection as well $[38,39]$. Interestingly, the presence of $P$. micra has also been strongly associated with multiple types of cancer. Numerous reports have identified a major enrichment of $P$. micra in colorectal tumors [5, 40-42], while others have reported an association of $P$. micra with oral and gastric cancers [4,

320 42, 43]. Given the substantial body of evidence for its role in human disease, the development of new genetic tools for P. micra is an essential step to reveal its unknown pathogenic mechanisms.

Here, we provide the first demonstration of $P$. micra natural competence. To the

325 best of our knowledge, this is not only a first for the species, but it is also likely the first report of natural competence within the entire Tissierellia class, which is a subgroup in the Firmicutes phylum largely comprised of microbial dark matter. The use of natural competence for DNA transformation has numerous advantages over artificial methods, such as chemical treatments (e.g., $\mathrm{CaCl}_{2}$ ) and

330 electroporation [44]. These approaches are also difficult to optimize for many fastidious anaerobes like $P$. micra, due to their sensitivity to excessive handling. Natural transformation can also be more efficient than those of artificial methods, as naturally transformed DNA enters the bacterial cytosol single-stranded [45]. Unlike duplex DNA introduced by artificial means, single-stranded DNA (ssDNA)

335 is largely resistant to degradation by most bacterial restriction enzymes [46, 47]. Furthermore, ssDNA can serve as a more efficient substrate for homologous recombination compared to duplex DNA. The recombinase RecA is specifically activated by the presence of ssDNA to form pre-synaptic filaments, which in turn, promote RecA to scan for homologous sequences, induce synapse formation, and

340 ultimately mediate recombination [48]. In our study, all of the P. micra isolates tested displayed some level of natural competence (Figure 2C and Figure 3B), though it is currently unclear why some strains exhibited higher transformation rates than others. A number of factors could contribute to these disparities such as strain-specific differences in the expression of competence related genes, variable

345 responses to competence-specific environmental cues, and/or an altered expression of transformation-limiting bacterial factors (e.g. nucleases) [49]. Our results demonstrate that some of these limiting factors can be mitigated by increasing the lengths of the homologous fragments contained on the mutagenesis constructs (Figure 3B) [50]. Based on this, we speculate that endogenous exonuclease 350 activity is likely to be a major determinant of the transformation efficiency 
observed with linear DNA constructs. However, it is also possible that longer segments of homology simply lead to higher rates of recombination.

After observing robust natural competence in $P$. micra, we examined the

355 feasibility of creating $\operatorname{rec} A$ deletion mutants. We chose $r e c A$ as a test case due to its anticipated and easily measured phenotypes. As expected, the rec $A$ mutant exhibited a total loss of detectable natural transformability as well as a MMCsensitive phenotype (Figure 4C and D). We were also able to complement these phenotypes by employing allelic exchange to insert the recA ORF immediately

360 downstream of the constitutively expressed tuf gene, creating an artificial tuf-rec $A$ operon (Figure 4B). This further illustrates a straightforward approach for genetic complementation without requiring a shuttle vector.

The subsequent expression of luciferase in $P$. micra led us to consider its use as a 365 reporter for the development of a regulated gene expression system. Previously, we developed a highly efficient xylose induction system $(\mathrm{XylR} / \mathrm{O})$ that functioned in several oral Streptococcus species [31]. Repressor/operator-based systems such as XylR/O (xylose) and LacR/O (IPTG) are contingent upon the uptake of inducer molecules as well as the heterologous expression of the corresponding repressor

370 proteins. These can be of limited utility for bacterial species in which repressor protein expression or inducer permeability may be limiting factors. Indeed, our attempts to develop a XylR/O system in $P$. micra were largely unsuccessful. This led us to consider an alternative approach using a riboswitch-based regulatory system for posttranscriptional control of gene expression. Using a synthetic

375 theophylline riboswitch [32], we demonstrated the potential utility of theophylline induction in $P$. micra, achieving up to a $\sim 9$-fold maximum induction of luciferase activity (Figure 6). In an attempt to further improve upon its performance, we introduced a series of riboswitch point mutations that were predicted to create a Rho-independent terminator-like structure in the absence of theophylline. Further

380 studies would be required to confirm whether the mutant riboswitch truly functions in termination. Regardless, these mutations were able to further increase the dynamic range of the theophylline riboswitch by nearly an order of magnitude (Figure 6). This new version that we now refer to as the Theo+ riboswitch, yielded low basal expression, while providing robust and tunable inducibility. In addition 385 to genetic complementation, this induction system is likely applicable for studies of both essential and toxic genes. It may also be useful for controlling target transcript levels and the timing of gene expression during infection studies.

Tn-seq is a powerful forward genetic screening tool used for genome-level 390 assessments of genetic fitness. Based upon the efficacy of P. micra natural transformation, we predicted that it would be well suited for in vitro Marinerbased transposon mutagenesis. Considering that the Himar transposase specifically targets TA dinucleotides [35], the high A+T content of the P. micra genome 
$(>70 \% \mathrm{~A}+\mathrm{T})$ makes it especially well-suited for Tn-seq analysis with

395 Himar/Mariner. The availability of a high efficiency natural competence protocol also circumvents the requirement for complex in vivo transposition methods employing conjugation, temperature sensitive plasmids, and ectopically expressed transposases. Based upon the results shown in figures $7 \mathrm{C}$ and $\mathrm{D}$, the current protocol should easily achieve the mutagenesis thresholds required for high400 density Tn-seq analysis. Thus, when combined with the other genetic tools described in this study, one can now reliably perform nearly all of the molecular genetic approaches required to characterize $P$. micra pathobiology. As such, $P$. micra should also be added to the list of genetically tractable oral microbes. 


\section{Materials and Methods}

\section{Isolation of Parvimonas from Clinical Odontogenic Abscess Samples}

Clinical sample collection and strain isolation protocols were reviewed by the Oregon Health and Science University Institutional Review Board (IRB) prior to 415 the initiation of the study and deemed to be not human subject research. All clinical specimens were collected by clinicians in the OHSU Pediatric Dental clinic using materials generated during routine treatment procedures. Specimens were deidentified of all Protected Health Information (PHI) as part of the collection protocol. Clinical specimens were derived from a pediatric cohort

420 undergoing tooth extraction due to odontogenic abscesses.

Briefly, abscess samples were collected using Dacron swabs and placed in prereduced transport media [21]. Abscess samples were vortexed and plated onto PMM agar media [12]. Plates were incubated in an anaerobic chamber $\left(85 \% \mathrm{~N}_{2}\right.$,

$42510 \% \mathrm{CO}_{2}, 5 \% \mathrm{H}_{2}$ ) at $37^{\circ} \mathrm{C}$ for 4 to 7 days. Bacterial colonies producing a black precipitate were passaged on fresh PMM plates. Candidate isolates were imaged by microscopy for the expected cellular morphologies and then screened by PCR using the primers $16 \mathrm{SFOR} / \mathrm{REV}$. Sequence identification of $16 \mathrm{~S}$ rRNA was confirmed using the expanded Human Oral Microbiome Database

430 (www.homd.org) [51].

\section{Bacterial Strains and Growth Conditions}

Parvimonas micra cultures were maintained in supplemented Brain Heart Infusion

435 medium (sBHI), which consisted of base Brain Heart Infusion medium (BHI; Gibco, Gaithersburg, MD) containing $0.5 \% \mathrm{wt} / \mathrm{vol}$ yeast extract (Fisher, Fair Lawn, $\mathrm{NJ}$ ), $0.005 \% \mathrm{wt} / \mathrm{vol}$ hemin (Sigma, St. Louis, MO), 0.001\% wt/vol menadione (Sigma, St. Louis, MO), and 0.05\% wt/vol cysteine (Sigma, St. Louis, MO). sBHI media and cultures were maintained in anaerobic conditions $\left(85 \% \mathrm{~N}_{2}, 10 \% \mathrm{CO}_{2}\right.$,

$\left.4405 \% \mathrm{H}_{2}\right)$ at $37^{\circ} \mathrm{C}$. For antibiotic selection, sBHI agar plates were supplemented with the following: rifampicin $\left(5 \mathrm{mg} \mathrm{L}^{-1}\right)$, kanamycin $\left(300 \mathrm{mg} \mathrm{L}^{-1}\right)$, and erythromycin $\left(15 \mathrm{mg} \mathrm{L}^{-1}\right)$.

To isolate spontaneous rifampicin resistant mutants, the $P$. micra reference strain ATCC 33270 was plated on $\mathrm{SBHI}$ agar containing rifampicin $\left(5 \mathrm{mg} \mathrm{L}^{-1}\right)$. A

445 rifampicin resistant mutant $\left(\right.$ rif $^{\mathrm{R}}$ ) was screened by PCR using the primers rpoB$1240 \mathrm{FOR}$ and rpoB-2095REV. Sequence analysis of this conserved region of rроB confirmed an A-to- $\mathrm{T}$ transversion resulting in an aspartic acid to valine 
substitution at amino acid 501 of RpoB.

\section{Electron Microscopy}

Bacteria were grown overnight in sBHI on ITO coverslips and fixed in $2.5 \%$ glutaraldehyde, $2.5 \%$ formaldehyde in sodium cacodylate buffer ( $\mathrm{pH} \mathrm{7.4).}$ Samples were rinsed in buffer, post-fixed in $2 \%$ osmium tetroxide for 30 minutes, rinsed in water, dehydrated in a graded ethanol series, and critical point dried in a Leica CPD300. Samples were mounted on aluminum stubs with a carbon tab and coated with $8 \mathrm{~nm}$ carbon using a Leica ACE600 coater. Imaging was performed on a ThermoFisher Scientific Helios NanoLabG3 DualBeam scanning electron microscope.

\section{Extraction of genomic DNA from $P$. micra}

Genomic DNA (gDNA) was purified using a phenol-chloroform extraction procedure [52]. Bacteria were harvested from sBHI agar plates, suspended in STES buffer (0.5M NaCl, 0.02 M EDTA, 0.2 M Tris-HCl (pH 8), $20 \mathrm{mg} \mathrm{mL}^{-1}$ lysozyme) and incubated at $55^{\circ} \mathrm{C}$ for $2 \mathrm{~h}$. SDS was added to a final concentration

465 of $1 \%$ followed by the addition of proteinase $\mathrm{K}(0.1 \mathrm{mg} / \mathrm{mL})$ and incubation at $55^{\circ} \mathrm{C}$ for $1 \mathrm{~h}$. One volume of phenol:chloroform:isoamyl alcohol (25:24:1) (Fisher, Fair Lawn, NJ) was added to the suspension and mixed gently. After centrifugation at $16,000 \times \mathrm{g}$, the aqueous layer was collected and treated with RNase $\left(50 \mu \mathrm{g} \mathrm{mL}^{-1}\right)$ at $37^{\circ} \mathrm{C}$ for $2 \mathrm{~h}$ followed by a second extraction with 470 phenol:chloroform:isoamyl alcohol. A 1:10 volume of $3 \mathrm{M}$ sodium acetate ( $\mathrm{pH}$ 5.5) and 3 volumes of ice-cold ethanol was added and incubated at $-20^{\circ} \mathrm{C}$ for $24 \mathrm{~h}$. DNA was pelleted by centrifugation at $16,000 \mathrm{xg}$, at $4^{\circ} \mathrm{C}$ for $30 \mathrm{~min}$ and subsequently washed with ice-cold ethanol (70\%). The DNA pellet was dried in a laminar flow hood and resuspended in $\mathrm{ddH}_{2} \mathrm{O}$.

\section{DNA transformation experiments}

Transformation assays were performed similarly as previously described [24]. Briefly, P. micra was grown on sBHI agar for $72 \mathrm{~h}$. Cells were harvested from plates and suspended into liquid sBHI and adjusted to an optical density $\mathrm{OD}_{600}$ of 480 0.4. Unless otherwise indicated, a total of $1 \mu \mathrm{g}$ of gDNA (dissolved in $20 \mu \mathrm{L}$ of $\mathrm{ddH}_{2} \mathrm{O}$ ) was spotted on sBHI agar plates. After complete absorption of the gDNA into the agar, $20 \mu \mathrm{L}$ of each bacteria suspension was pipetted over the DNA spots and absorbed. Plates were incubated in anaerobic conditions at $37^{\circ} \mathrm{C}$ for $24 \mathrm{~h}$. 
Following the incubation period, cells were then harvested into $50 \mu \mathrm{L}$ of sBHI and vortexed. Serial dilutions were spread onto sBHI agar plates containing the appropriate antibiotic as well as on nonselective sBHI plates to determine total CFU counts. Transformation efficiency was calculated as the ratio of antibiotic resistant transformants to total CFU. For allelic replacement mutagenesis and genetic complementation, transformations were performed similarly as described 490 above.

\section{P. micra ermB insertion mutagenesis constructs}

Primers and mutagenesis constructs were designed using Serial Cloner software (https://serial-cloner.en.softonic.com). All primers used in this study are listed in

495 Table S1. All PCR amplifications were performed using Phusion High-Fidelity Polymerase (Fisher, Fair Lawn, NJ). For insertion mutagenesis constructs, an erythromycin resistance cassette $(\mathrm{erm} B)$ was inserted directly downstream of the tuf gene. The ermB cassette was amplified using primers ermR-FOR/REV and the plasmid pJY4164 as a PCR template [25]. Upstream homologous regions were

500 generated using the primer pair tuf-FOR/REV for all isolates. For downstream homologous regions, the primer pair down-tufFOR/REV was used with isolates A1, A3, and A28, while down-tufFORa/tuf-REV was used for strains A11 and ATCC 33270. gDNA from each respective $P$. micra strain was used as the PCR template. All amplicons were screened for size, column purified (Qiagen,

505 Germantown, MD), and assembled using Gibson Assembly Master Mix (NEB, Ipswich, MA) as per manufacturer's instructions. The assembled construct was amplified by PCR using the primer pair tuf-FOR/down-tuf-REV, screened for size, and column purified. Transformation assays using these constructs were performed as described above. Primer pair tuf-FOR*/down-tuf-REV* were used to

510 screen select transformants for ermB insertion.

To generate $e r m B$ insertion constructs with flanking regions of varying sizes, gDNA from an ermB insertion mutant (ermR A28) was used as a template. The primer pairs tuf-250-FOR/REV, tuf-FOR/ down-tuf-REV, tuf-1750-FOR/REV, and tuf-2500-FOR/REV were used to generate homologous fragments of $250 \mathrm{bp}$,

$5151.0 \mathrm{~kb}, 1.75 \mathrm{~kb}$, and $2.5 \mathrm{~kb}$ respectively. For transformation assays, a molar equivalent to $1 \mu \mathrm{g}$ of the $1.0 \mathrm{~kb}$ homologous flank construct was used for all constructs. Transformation assays with these constructs were performed as described above.

\section{$520 \quad P$. micra rec $A$ deletion and genetic complementation}

For construction of the rec $A$ deletion mutant, an ermB cassette flanked by 
homologous upstream and downstream regions to $\operatorname{rec} A$ was assembled. The erm $B$ cassette was amplified using the primer pair ermR-FOR/REV and pJY4164 as the PCR template. Upstream and downstream homologous regions to recA were

525 generated using the primer pairs up-recA-FOR/REV and down-recA-FOR/REV respectively, with strain A28 gDNA used as the PCR template. All amplicons were screened for size, column purified, and assembled using Gibson Assembly Master Mix. The assembled construct was amplified by PCR using the primer pair up-recA-FOR/down-recA-REV, screened for size, and column purified.

530 Transformants were selected on agar plates supplemented with erythromycin.

To generate the $\operatorname{rec} A$ knock-in strain, a construct was made containing the $\operatorname{rec} A$ ORF along with a kanamycin resistance cassette aphAIII inserted immediately downstream of the tuf gene. PCR fragments homologous to tuf and its downstream sequence were generated using the primer pairs tuf-FOR/tuf-REVa and down-tuf-

$535 \mathrm{FORb} /$ tuf-2500-REV respectively, with A28 gDNA serving as the PCR template. The $r e c A$ gene was amplified using the primer pair recA-FOR/REV and A28 gDNA as the template. The kanamycin resistance cassette aphAIII was amplified using the primer pair kan-FOR/REV with plasmid pWVTK as the template [25]. All amplicons were screened for size, column purified, and assembled using

540 Gibson Assembly Master Mix. The assembled construct was PCR amplified using the primer pair tuf-FOR/tuf-2500-REV and then column purified. Transformants were selected on agar plates supplemented with kanamycin. A complemented $\Delta r e c A$ mutant strain was generated by transforming the $r e c A$ knock-in strain with the rec $A$ deletion construct described above. Transformants were selected on agar

545 plates supplemented with kanamycin and erythromycin.

To compare transformation efficiencies of the various rec $A$ mutants, PCR amplicons were generated with the primer pair rpoB-1240FOR/2095REV using gDNA from the spontaneous $P$. micra rifampicin resistant strain described above. PCR products were screened for size and column purified. Transformation assays

550 were performed as described above using $\mathrm{sBHI} \pm$ rifampicin. For mitomycin $\mathrm{C}$ (Sigma, St. Louis, MO) sensitivity experiments, each strain was suspended in $\mathrm{sBHI}$ at an $\mathrm{OD}_{600}$ of 1.0 and then serial dilutions were plated on $\mathrm{sBHI}$ agar plates containing varying dosages of $\mathrm{MMC}\left(0 \mu \mathrm{g} \mathrm{mL}^{-1}, 0.5 \mu \mathrm{g} \mathrm{mL}^{-1}, 1 \mu \mathrm{g} \mathrm{mL}^{-1}, 2 \mu \mathrm{g} \mathrm{mL}\right.$ ${ }^{1}$, and $\left.4 \mu \mathrm{g} \mathrm{mL}{ }^{-1}\right)$. Plates were incubated in an anaerobic chamber at $37^{\circ} \mathrm{C}$ for $72 \mathrm{~h}$.

\section{Green renilla luciferase expression in $P$. micra}

To express green renilla luciferase in $P$. micra, a construct was assembled containing the ren $G$ ORF along with a kanamycin resistance cassette aphAIII inserted immediately downstream of the tuf gene. PCR fragments homologous to

560 tuf and its downstream sequence were generated using the primer pairs tuf-2500- 
FOR/tuf-REVa and down-tuf-FORb/tuf-2500-REV respectively, with A28 gDNA as the template. A PCR fragment of $\operatorname{ren} G$ was generated using the primer pair renG-RBS-FOR/renG-REV with gDNA from strain brsRM-renG as the template [25]. The kanamycin resistance cassette aphAIII was amplified using the primer

565 pair kan-FOR/REV and plasmid pWVTK as the template. All amplicons were screened for size, column purified, and assembled using Gibson Assembly Master Mix. The assembled construct was amplified by PCR using the primer pair tuf2500-FOR/REV, screened for size, and column purified. The amplicon was transformed into wild-type P. micra strain A28 and the transformants selected on 570 agar plates supplemented with kanamycin.

\section{Construction of $\boldsymbol{P}$. micra inducible luciferase strain}

For inducible expression of renilla luciferase in $P$. micra, a construct containing a $r p o B$ promoter and riboswitch preceding $\operatorname{ren} G$ followed by a kanamycin cassette

575 was inserted directly downstream of the tuf gene rho-independent terminator. PCR fragments homologous to tuf, including its terminator and its downstream sequence were generated using the primer pairs tuf-2500-FOR/tuf-53-REV and down-tuf-FORb/tuf-2500-REV respectively, using strain A28 gDNA as the template. The riboswitch regulatory region was PCR amplified with the primer

580 pair ribo-FOR/REV using a synthetic riboswitch DNA fragment (IDT, Coralville, Iowa) as the template (see Table S1). The $\operatorname{ren} G$ gene was amplified with primer pair renG-FOR/REV using gDNA from the strain brsRM-renG. The kanamycin resistance cassette was amplified using the primer pair kan-FOR/REV and plasmid pWVTK as a template. All amplicons were screened for size, column purified, and 585 assembled using Gibson Assembly Master Mix. The resulting construct was amplified by PCR using the primer pair tuf-2500-FOR/REV, screened for size, and column purified. This product was transformed into wild-type $P$. micra isolate A28 and transformants selected on agar plates supplemented with kanamycin.

For the Theo+ riboswitch variant, a construct was generated using the primer pairs 590 tuf-2500-FOR/ theo-plus-ribo-REV and theo-plus-renG-FOR/tuf-2500REV using gDNA from the inducible mutant described above. PCR products were assembled using Gibson Assembly Master mix and amplified using the primer pair tuf-2500FOR/REV. Transformants were selected on sBHI agar plates containing kanamycin.

\section{Luciferase assays}

RenG expression strains were grown for $72 \mathrm{~h}$ on sBHI agar plates supplemented 
with kanamycin. Bacteria were suspended in liquid sBHI media with a range of theophylline concentrations ( $0 \mathrm{mM}, 0.0078 \mathrm{mM}, 0.0156 \mathrm{mM}, 0.03125 \mathrm{mM}$,

$600 \quad 0.0625 \mathrm{mM}, 0.125 \mathrm{mM}, 0.25 \mathrm{mM}, 0.5 \mathrm{mM}, 1 \mathrm{mM}, 2 \mathrm{mM}, 4 \mathrm{mM}$, and $8 \mathrm{mM})$ at an $\mathrm{OD}_{600}$ of 0.1 and then incubated in an anaerobic chamber at $37^{\circ} \mathrm{C}$ for $20 \mathrm{~h}$. Coelenterazine-h solution (Prolume, Pinetop, AZ) was added to each sample (3.4 $\mu \mathrm{g} \mathrm{mL} \mathrm{m}^{-1}$ ) and luciferase activity was measured on a Promega Glomax Discover luminometer. Optical densities were measured immediately after measuring

605 luciferase activity for normalization. Normalized activity was expressed as relative light units (RLU), which is the luminescence value/OD 600 .

\section{Generation of a transposon insertion library in $P$. micra}

610 A transposon library was generated in P. micra strain A28 using a previously described protocol [33]. Briefly, in vitro transposon mutagenesis was performed by combining $1 \mu \mathrm{g}$ of gDNA from isolate A28, $1 \mu \mathrm{g}$ of the Mariner transposoncontaining vector pT-MGL-erm [25], and varying amounts of the MarC9 transposase $(0 \mu \mathrm{g}, 0.125 \mu \mathrm{g}, 0.25 \mu \mathrm{g}, 0.5 \mu \mathrm{g}$, and $1 \mu \mathrm{g})$. The mixture was

615 incubated for $30^{\circ} \mathrm{C}$ for $1 \mathrm{~h}$ followed by a $75^{\circ} \mathrm{C}$ incubation for $10 \mathrm{~min}$ and then incubation on ice. The DNA was precipitated with ethanol and transposon junctions subsequently repaired. The resulting transposon reactions were transformed directly into P. micra isolate A28. Serial dilutions were plated on nonselective sBHI agar plates for total counts, while transposon mutants were

620 selected on sBHI agar plates supplemented with erythromycin.

\section{Statistical analysis}

All statistical analyses were performed using GraphPad Prism software to 625 calculate significance via two-tailed Student's $t$-test. Statistical significance was assessed using a cutoff value of $\mathrm{P}<0.05$. 


\section{Acknowledgments}

We are grateful to members of the Merritt laboratory for valuable insights regarding this manuscript. We thank Laura Higashi for help with scientific illustration. Images were prepared using Procreate, BioRender.com, Adobe

635 Illustrator, and GraphPad Prism. Electron microscopy was performed at the Multiscale Microscopy Core, a member of the OHSU University Shared Resource Cores. This work was supported by NIH-NIDCR grants DE029612 and DE029492 awarded to J.K. and DE028252 awarded to J.M. 


\section{References}

1. Tindall BJ, Euzeby JP. Proposal of Parvimonas gen. nov. and Quatrionicoccus gen. nov. as replacements for the illegitimate, prokaryotic, generic names Micromonas

645 Murdoch and Shah 2000 and Quadricoccus Maszenan et al. 2002, respectively. Int J Syst Evol Microbiol. 2006;56(Pt 11):2711-3. Epub 2006/11/04. doi: 10.1099/ijs.0.64338-0. PubMed PMID: 17082417.

2. Murdoch DAS, H.N. Reclassification of Peptostreptococcus magnus (Prevot 1933) Holdeman and Moore 1972 as Finegoldia magna comb. nov. and

650 Peptostreptococcus micros (Prevot 1933) Smith 1957 as Micromonas micros comb. nov. Anaerobe. 1999;5(5):555-9. doi: https://doi.org/10.1006/anae.1999.0197.

3. Murphy EC, Frick IM. Gram-positive anaerobic cocci--commensals and opportunistic pathogens. FEMS Microbiol Rev. 2013;37(4):520-53. Epub 2012/10/04. doi: 10.1111/1574-6976.12005. PubMed PMID: 23030831.

655 4. Coker OO, Dai Z, Nie Y, Zhao G, Cao L, Nakatsu G, et al. Mucosal microbiome dysbiosis in gastric carcinogenesis. Gut. 2018;67(6):1024-32. Epub 2017/08/03. doi: 10.1136/gutjnl-2017-314281. PubMed PMID: 28765474; PubMed Central PMCID: PMCPMC5969346.

5. Flemer B, Warren RD, Barrett MP, Cisek K, Das A, Jeffery IB, et al. The oral 660 microbiota in colorectal cancer is distinctive and predictive. Gut. 2018;67(8):1454-63. Epub 2017/10/11. doi: 10.1136/gutjnl-2017-314814. PubMed PMID: 28988196; PubMed Central PMCID: PMCPMC6204958.

6. Saffarian A, Mulet C, Regnault B, Amiot A, Tran-Van-Nhieu J, Ravel J, et al. Crypt- and Mucosa-Associated Core Microbiotas in Humans and Their Alteration in

665 Colon Cancer Patients. mBio. 2019;10(4). Epub 2019/07/18. doi: 10.1128/mBio.0131519. PubMed PMID: 31311881 ; PubMed Central PMCID: PMCPMC6635529.

7. Watanabe T, Hara Y, Yoshimi Y, Fujita Y, Yokoe M, Noguchi Y. Clinical characteristics of bloodstream infection by Parvimonas micra: retrospective case series and literature review. BMC Infect Dis. 2020;20(1):578. Epub 2020/08/08. doi:

670 10.1186/s12879-020-05305-y. PubMed PMID: 32758181; PubMed Central PMCID: PMCPMC7405351.

8. Yang CY, Yeh YM, Yu HY, Chin CY, Hsu CW, Liu H, et al. Oral Microbiota Community Dynamics Associated With Oral Squamous Cell Carcinoma Staging. Front Microbiol. 2018;9:862. Epub 2018/05/19. doi: 10.3389/fmicb.2018.00862. PubMed

675 PMID: 29774014; PubMed Central PMCID: PMCPMC5943489.

9. Yao Y, Ni H, Wang X, Xu Q, Zhang J, Jiang L, et al. A New Biomarker of Fecal Bacteria for Non-Invasive Diagnosis of Colorectal Cancer. Front Cell Infect Microbiol. 2021;11:744049. Epub 2022/01/04. doi: 10.3389/fcimb.2021.744049. PubMed PMID: 34976850; PubMed Central PMCID: PMCPMC8719628.

680 10. Colombo AP, Boches SK, Cotton SL, Goodson JM, Kent R, Haffajee AD, et al. Comparisons of subgingival microbial profiles of refractory periodontitis, severe periodontitis, and periodontal health using the human oral microbe identification microarray. J Periodontol. 2009;80(9):1421-32. Epub 2009/09/03. doi: 10.1902/jop.2009.090185. PubMed PMID: 19722792; PubMed Central PMCID: 685 PMCPMC3627366. 
11. Nonnenmacher C, Dalpke A, Mutters R, Heeg K. Quantitative detection of periodontopathogens by real-time PCR. J Microbiol Methods. 2004;59(1):117-25. Epub 2004/08/25. doi: 10.1016/j.mimet.2004.06.006. PubMed PMID: 15325758.

12. Turng BF, Guthmiller JM, Minah GE, Falkler WA, Jr. Development and

690 evaluation of a selective and differential medium for the primary isolation of Peptostreptococcus micros. Oral Microbiol Immunol. 1996;11(5):356-61. Epub 1996/10/01. doi: 10.1111/j.1399-302x.1996.tb00194.x. PubMed PMID: 9028263. 13. Rocas IN, Siqueira JF, Jr. Root canal microbiota of teeth with chronic apical periodontitis. J Clin Microbiol. 2008;46(11):3599-606. Epub 2008/09/05. doi:

695 10.1128/JCM.00431-08. PubMed PMID: 18768651; PubMed Central PMCID: PMCPMC2576597.

14. Gomes BP, Berber VB, Kokaras AS, Chen T, Paster BJ. Microbiomes of Endodontic-Periodontal Lesions before and after Chemomechanical Preparation. J Endod. 2015;41(12):1975-84. Epub 2015/11/02. doi: 10.1016/j.joen.2015.08.022.

700 PubMed PMID: 26521147; PubMed Central PMCID: PMCPMC7061340.

15. Siqueira JF, Jr., Rocas IN. Microbiology and treatment of acute apical abscesses. Clin Microbiol Rev. 2013;26(2):255-73. Epub 2013/04/05. doi: 10.1128/CMR.00082-12. PubMed PMID: 23554416; PubMed Central PMCID: PMCPMC3623375.

16. Neilands J, Davies JR, Bikker FJ, Svensater G. Parvimonas micra stimulates

705 expression of gingipains from Porphyromonas gingivalis in multi-species communities. Anaerobe. 2019;55:54-60. Epub 2018/10/26. doi: 10.1016/j.anaerobe.2018.10.007. PubMed PMID: 30359695.

17. van Dalen PJ, van Deutekom-Mulder EC, de Graaff J, van Steenbergen TJ. Pathogenicity of Peptostreptococcus micros morphotypes and Prevotella species in pure 710 and mixed culture. J Med Microbiol. 1998;47(2):135-40. Epub 1999/01/08. doi: 10.1099/00222615-47-2-135. PubMed PMID: 9879956.

18. Cogoni V, Morgan-Smith A, Fenno JC, Jenkinson HF, Dymock D. Treponema denticola chymotrypsin-like proteinase (CTLP) integrates spirochaetes within oral microbial communities. Microbiology (Reading). 2012;158(Pt 3):759-70. Epub

715 2012/02/09. doi: 10.1099/mic.0.055939-0. PubMed PMID: 22313692; PubMed Central PMCID: PMCPMC4851253.

19. Horiuchi A, Kokubu E, Warita T, Ishihara K. Synergistic biofilm formation by Parvimonas micra and Fusobacterium nucleatum. Anaerobe. 2020;62:102100. Epub 2019/09/16. doi: 10.1016/j.anaerobe.2019.102100. PubMed PMID: 31521732.

720 20. Liu K, Hou BX. The regulation of DLTA gene in bacterial growth and biofilm formation by Parvimonas micra. Eur Rev Med Pharmacol Sci. 2018;22(13):4033-44. Epub 2018/07/20. doi: 10.26355/eurrev_201807_15390. PubMed PMID: 30024592. 21. Doan N, Contreras A, Flynn J, Morrison J, Slots J. Proficiencies of three anaerobic culture systems for recovering periodontal pathogenic bacteria. J Clin

725 Microbiol. 1999;37(1):171-4. Epub 1998/12/17. doi: 10.1128/JCM.37.1.171-174.1999. PubMed PMID: 9854085; PubMed Central PMCID: PMCPMC84198.

22. Jin DJ, Gross CA. Mapping and sequencing of mutations in the Escherichia coli rpoB gene that lead to rifampicin resistance. J Mol Biol. 1988;202(1):45-58. Epub 1988/07/05. doi: 10.1016/0022-2836(88)90517-7. PubMed PMID: 3050121.

730 23. Telenti A, Imboden P, Marchesi F, Lowrie D, Cole S, Colston MJ, et al. Detection of rifampicin-resistance mutations in Mycobacterium tuberculosis. Lancet. 
1993;341(8846):647-50. Epub 1993/03/13. doi: 10.1016/0140-6736(93)90417-f. PubMed PMID: 8095569.

24. Knapp S, Brodal C, Peterson J, Qi F, Kreth J, Merritt J. Natural Competence Is

735 Common among Clinical Isolates of Veillonella parvula and Is Useful for Genetic Manipulation of This Key Member of the Oral Microbiome. Front Cell Infect Microbiol. 2017;7:139. Epub 2017/05/06. doi: 10.3389/fcimb.2017.00139. PubMed PMID: 28473967; PubMed Central PMCID: PMCPMC5397411.

25. Zou Z, Qin H, Brenner AE, Raghavan R, Millar JA, Gu Q, et al. LytTR

740 Regulatory Systems: A potential new class of prokaryotic sensory system. PLoS Genet. 2018;14(10):e1007709. Epub 2018/10/09. doi: 10.1371/journal.pgen.1007709. PubMed PMID: 30296267 ; PubMed Central PMCID: PMCPMC6193735.

26. Kung SH, Retchless AC, Kwan JY, Almeida RP. Effects of DNA size on transformation and recombination efficiencies in Xylella fastidiosa. Appl Environ

745 Microbiol. 2013;79(5):1712-7. Epub 2013/01/15. doi: 10.1128/AEM.03525-12. PubMed PMID: 23315739; PubMed Central PMCID: PMCPMC3591940.

27. Shen P, Huang HV. Homologous recombination in Escherichia coli: dependence on substrate length and homology. Genetics. 1986;112(3):441-57. Epub 1986/03/01. doi: 10.1093/genetics/112.3.441. PubMed PMID: 3007275; PubMed Central PMCID:

$750 \quad$ PMCPMC1202756.

28. Clark AJ, Margulies AD. Isolation and Characterization of RecombinationDeficient Mutants of Escherichia Coli K12. Proc Natl Acad Sci U S A. 1965;53:451-9. Epub 1965/02/01. doi: 10.1073/pnas.53.2.451. PubMed PMID: 14294081; PubMed Central PMCID: PMCPMC219534.

755 29. Lusetti SL, Wood EA, Fleming CD, Modica MJ, Korth J, Abbott L, et al. Cterminal deletions of the Escherichia coli RecA protein. Characterization of in vivo and in vitro effects. J Biol Chem. 2003;278(18):16372-80. Epub 2003/02/25. doi: 10.1074/jbc.M212917200. PubMed PMID: 12598539.

30. Merritt J, Senpuku H, Kreth J. Let there be bioluminescence: development of a

760 biophotonic imaging platform for in situ analyses of oral biofilms in animal models. Environ Microbiol. 2016;18(1):174-90. Epub 2015/06/30. doi: 10.1111/14622920.12953. PubMed PMID: 26119252; PubMed Central PMCID: PMCPMC5050008. 31. Xie Z, Qi F, Merritt J. Development of a tunable wide-range gene induction system useful for the study of streptococcal toxin-antitoxin systems. Appl Environ 765 Microbiol. 2013;79(20):6375-84. Epub 2013/08/13. doi: 10.1128/AEM.02320-13. PubMed PMID: 23934493; PubMed Central PMCID: PMCPMC3811191.

32. Topp S, Reynoso CM, Seeliger JC, Goldlust IS, Desai SK, Murat D, et al. Synthetic riboswitches that induce gene expression in diverse bacterial species. Appl Environ Microbiol. 2010;76(23):7881-4. Epub 2010/10/12. doi: 10.1128/AEM.01537-10.

770 PubMed PMID: 20935124; PubMed Central PMCID: PMCPMC2988590.

33. van Opijnen T, Lazinski DW, Camilli A. Genome-Wide Fitness and Genetic Interactions Determined by Tn-seq, a High-Throughput Massively Parallel Sequencing Method for Microorganisms. Curr Protoc Microbiol. 2015;36:1E 3 1-E 3 24. Epub 2015/02/03. doi: 10.1002/9780471729259.mc01e03s36. PubMed PMID: 25641100;

775 PubMed Central PMCID: PMCPMC4696536.

34. Ding Q, Tan KS. Himar1 Transposon for Efficient Random Mutagenesis in Aggregatibacter actinomycetemcomitans. Front Microbiol. 2017;8:1842. Epub 
2017/10/12. doi: 10.3389/fmicb.2017.01842. PubMed PMID: 29018421; PubMed Central PMCID: PMCPMC5622930.

780 35. Lampe DJ, Churchill ME, Robertson HM. A purified mariner transposase is sufficient to mediate transposition in vitro. EMBO J. 1996;15(19):5470-9. Epub 1996/10/01. PubMed PMID: 8895590; PubMed Central PMCID: PMCPMC452289. 36. Brook I. Recovery of anaerobic bacteria from clinical specimens in 12 years at two military hospitals. J Clin Microbiol. 1988;26(6):1181-8. Epub 1988/06/01. doi:

785 10.1128/jcm.26.6.1181-1188.1988. PubMed PMID: 3384929; PubMed Central PMCID: PMCPMC266558.

37. Murdoch DA, Mitchelmore IJ, Tabaqchali S. Peptostreptococcus micros in polymicrobial abscesses. Lancet. 1988;1(8585):594. Epub 1988/03/12. doi: 10.1016/s0140-6736(88)91393-1. PubMed PMID: 2894531.

790 38. Kim EY, Baek YH, Jung DS, Woo KS. Concomitant Liver and Brain Abscesses Caused by Parvimonas Micra. Korean J Gastroenterol. 2019;73(4):230-4. Epub 2019/04/30. doi: 10.4166/kjg.2019.73.4.230. PubMed PMID: 31030461.

39. Yoo LJH, Zulkifli MD, O'Connor M, Waldron R. Parvimonas micra spondylodiscitis with psoas abscess. BMJ Case Rep. 2019;12(11). Epub 2019/11/22. doi:

795 10.1136/bcr-2019-232040. PubMed PMID: 31748364; PubMed Central PMCID: PMCPMC6887430.

40. Drewes JL, White JR, Dejea CM, Fathi P, Iyadorai T, Vadivelu J, et al. Highresolution bacterial 16S rRNA gene profile meta-analysis and biofilm status reveal common colorectal cancer consortia. NPJ Biofilms Microbiomes. 2017;3:34. Epub

800 2017/12/08. doi: 10.1038/s41522-017-0040-3. PubMed PMID: 29214046; PubMed Central PMCID: PMCPMC5707393.

41. Purcell RV, Visnovska M, Biggs PJ, Schmeier S, Frizelle FA. Distinct gut microbiome patterns associate with consensus molecular subtypes of colorectal cancer. Sci Rep. 2017;7(1):11590. Epub 2017/09/16. doi: 10.1038/s41598-017-11237-6. PubMed

805 PMID: 28912574; PubMed Central PMCID: PMCPMC5599497.

42. Zhang M, Lv Y, Hou S, Liu Y, Wang Y, Wan X. Differential Mucosal Microbiome Profiles across Stages of Human Colorectal Cancer. Life (Basel). 2021;11(8). Epub 2021/08/28. doi: 10.3390/life11080831. PubMed PMID: 34440574; PubMed Central PMCID: PMCPMC8401903.

810 43. Sun J, Tang Q, Yu S, Xie M, Xie Y, Chen G, et al. Role of the oral microbiota in cancer evolution and progression. Cancer Med. 2020;9(17):6306-21. Epub 2020/07/09. doi: 10.1002/cam4.3206. PubMed PMID: 32638533; PubMed Central PMCID: PMCPMC7476822.

44. Dubnau D, Blokesch M. Mechanisms of DNA Uptake by Naturally Competent

815 Bacteria. Annu Rev Genet. 2019;53:217-37. Epub 2019/08/23. doi: 10.1146/annurevgenet-112618-043641. PubMed PMID: 31433955.

45. Chen I, Dubnau D. DNA uptake during bacterial transformation. Nat Rev Microbiol. 2004;2(3):241-9. Epub 2004/04/15. doi: 10.1038/nrmicro844. PubMed PMID: 15083159 .

820 46. Blakesley RW, Dodgson JB, Nes IF, Wells RD. Duplex regions in "singlestranded" phiX174 DNA are cleaved by a restriction endonuclease from Haemophilus aegyptius. J Biol Chem. 1977;252(20):7300-6. Epub 1977/10/25. PubMed PMID: 71298. 
47. Boyer HW. Restriction and modification of DNA: enzymes and substrates. Introductory remarks. Fed Proc. 1974;33(5):1125-7. Epub 1974/05/01. PubMed PMID:

8254599005.

48. Renkawitz J, Lademann CA, Jentsch S. Mechanisms and principles of homology search during recombination. Nat Rev Mol Cell Biol. 2014;15(6):369-83. Epub 2014/05/16. doi: 10.1038/nrm3805. PubMed PMID: 24824069.

49. Huang M, Liu M, Huang L, Wang M, Jia R, Zhu D, et al. The activation and

830 limitation of the bacterial natural transformation system: The function in genome evolution and stability. Microbiol Res. 2021;252:126856. Epub 2021/08/29. doi: 10.1016/j.micres.2021.126856. PubMed PMID: 34454311.

50. Dutra BE, Sutera VA, Jr., Lovett ST. RecA-independent recombination is efficient but limited by exonucleases. Proc Natl Acad Sci U S A. 2007;104(1):216-21.

835 Epub 2006/12/22. doi: 10.1073/pnas.0608293104. PubMed PMID: 17182742; PubMed Central PMCID: PMCPMC1765439.

51. Escapa IF, Chen T, Huang Y, Gajare P, Dewhirst FE, Lemon KP. New Insights into Human Nostril Microbiome from the Expanded Human Oral Microbiome Database (eHOMD): a Resource for the Microbiome of the Human Aerodigestive Tract.

840 mSystems. 2018;3(6). Epub 2018/12/12. doi: 10.1128/mSystems.00187-18. PubMed PMID: 30534599; PubMed Central PMCID: PMCPMC6280432.

52. Cho E, Park SN, Lim YK, Shin Y, Paek J, Hwang CH, et al. Fusobacterium hwasookii sp. nov., Isolated from a Human Periodontitis Lesion. Curr Microbiol. 2015;70(2):169-75. Epub 2014/09/27. doi: 10.1007/s00284-014-0692-7. PubMed PMID: 84525257648. 


\section{Figure legend}

850 Figure 1. Parvimonas micra clinical isolation scheme. (A) (i) Odontogenic abscess samples were collected into anaerobic transport media and (ii) grown on PMM selective/differential agar. Putative colonies were (iii) confirmed morphologically and (iv) submitted for $16 \mathrm{~S}$ rRNA sequencing to verify $P$. micra. (B) Wild-type strain ATCC 33270 was (i) grown on non-selective sBHI agar and

855 (ii) subsequently placed on rifampicin containing $\mathrm{sBHI}$ media to isolate a spontaneous rifampicin resistance strain $\left(\right.$ rif $\left.^{\mathrm{R}}\right)$. (iii) The $\operatorname{rpoB}$ gene was sequenced and (iv) genomic DNA was isolated from both the if $^{R}$ and wild type (wt) rifampicin sensitive $\left(\right.$ (rif $\left.^{\mathrm{S}}\right)$ strain. (C) The deduced amino acid sequences of the wild-type rif $^{S}$ and rif $^{R}$ RpoB proteins are shown. The rif $^{R}$ mutation is shown in 860 red font.

Figure 2. Insertion of resistance cassette into $\boldsymbol{P}$. micra clinical isolates. (A) Mutagenesis construct (top) was inserted into the P. micra chromosome through homologous recombination (middle) yielding erythromycin resistant transformants

865 (bottom). The primers used for PCR verification of genotypes are illustrated by red arrows. (B) Insertion of the erythromycin resistance cassette $(\sim 1 \mathrm{~kb})$ was confirmed by PCR for a selected transformant (ermR A28). (C) Transformation frequencies of $P$. micra wild-type strains. Transformation frequencies are expressed as the ratio of erythromycin resistant CFUs to total CFUs. Values

870 represent the averages from triplicate independent determinations \pm SD. "n.d." indicates a transformation efficiency below the detection limit of the assay.

Figure 3. Comparison of homology vs. transformation efficiency in P. micra. (A) Mutagenesis constructs were generated with flanking regions of various sizes.

875 (B) Wild-type clinical isolates A28 and A1 were transformed with the mutagenesis constructs illustrated in panel A. Values are averaged from triplicate independent determinations \pm SD. "n.d." indicates a transformation efficiency below the limit of detection of the assay.

880 Figure 4. Targeted mutations of $\boldsymbol{r e c} \boldsymbol{A}$ in $\boldsymbol{P}$. micra. (A) The recA deletion construct (top) that was inserted into the P. micra A28 chromosome through homologous recombination (middle), resulting in allelic replacement of the recA ORF with an $e r m B$ cassette (bottom). (B) A recA knock-in mutagenesis construct (top) was inserted into the $P$. micra chromosome through homologous

885 recombination (middle), resulting in the insertion of the recA ORF and aphAIII cassette downstream of EF-Tu (bottom). The $\Delta r e c A$ mutation was complemented by moving this mutation into the rec $A$ knock-in mutant background. (C) Transformation frequency of wild-type A28 (WT), A28 $\Delta r e c A$ mutant $(\Delta \mathrm{A})$, double rec $A$-expressing knock-in mutant $(+\mathrm{A})$, and complemented $\Delta r e c A$ mutant $890(+\mathrm{A} / \triangle \mathrm{A})$. Transformation frequency is expressed as the ratio of rifampicin 
resistant bacteria to total CFUs. (D) Mitomycin C survival curves. All values are averaged from triplicate independent determinations \pm SD. For transformation assays, comparisons are indicated by brackets. For MMC experiments, comparisons are presented relative to $\Delta \mathrm{A}$. Statistical analysis was performed using 895 a two-tailed Student's $t$-test. “*” indicates a $\mathrm{p}<0.05$.

Figure 5. Green renilla luciferase (RenG) expression in $\boldsymbol{P}$. micra. (A) Genomic context and sequence of a constitutively expressed ren $G$ transcription fusion to the tuf gene. (B) Luciferase activity was measured in wild-type strain

900 A28 and the luciferase reporter strain (c-ren $G$ ). Normalized luciferase activity (RLU) was calculated by dividing raw luciferase values by the culture optical density $\mathrm{OD}_{600}$ values. (C) Correlation between viable cell counts (CFUs) and luciferase reporter values in the constitutive luciferase reporter strain c-ren $G$ $\left(\mathrm{R}^{2}=0.99\right)$. All data points represent the average values $\pm \mathrm{SD}$ from triplicate

905 independent determinations \pm SD. Statistical analysis was performed using a twotailed Student's $t$-test. " **" indicates a $\mathrm{p}<0.05$.

Figure 6. Regulated gene expression system in $\boldsymbol{P}$. micra. (A) Genomic context (top) and corresponding sequence (bottom) for an inducible luciferase reporter strain (i-ren $G$ ) in P. micra. The tuf gene Rho-independent terminator is indicated

910 by a ball and stick icon. The sequences are shown for the promoter region of the luciferase reporter, with the original theophylline riboswitch (underlined) and Theo + riboswitch (theo $+i-r e n G$ ) (dashed lines, bottom) indicated. Promoter elements are shown in red font, while the ribosomal binding site is in blue font. (B) The mFold webserver (http://www.unafold.org/mfold/applications/rna-

915 folding-form.php) was used to predict the secondary structures of the original (left image) and Theo+ (right image) theophylline riboswitches. (C) The original and Theo+ riboswitches were compared for their abilities to regulate luciferase reporter activity over a range of theophylline concentrations. Normalized luciferase activity (RLU) was calculated by dividing raw luciferase values by the

920 respective optical density $\mathrm{OD}_{600}$ values. Data points represent the average values $\pm \mathrm{SD}$ from triplicate independent determinations $\pm \mathrm{SD}$.

Figure 7. In vitro Mariner transposon mutagenesis in $\boldsymbol{P}$. micra. (A) Purification of recombinant MarC9 transposase and (B) the mini-Mariner transposon plasmid pT-MGL-erm. (C) $1 \mu \mathrm{g}$ of $P$. micra gDNA was mutagenized

925 using $0.125,0.25,0.5$, and $1.0 \mu \mathrm{g}$ of recombinant MarC9 transposase with a fixed quantity of the mini-Mariner transposon. These reactions were subsequently transformed into wild-type P. micra strain A28. The transformation results are shown on the graph. Data are expressed as the average number of transposon mutants/ $\mu \mathrm{g}$ of gDNA. Values are averaged from triplicate independent

930 determinations \pm SD. "n.d." indicates a transformation efficiency below the detection limit of the assay. 
Figure 1

A

i.

ii.
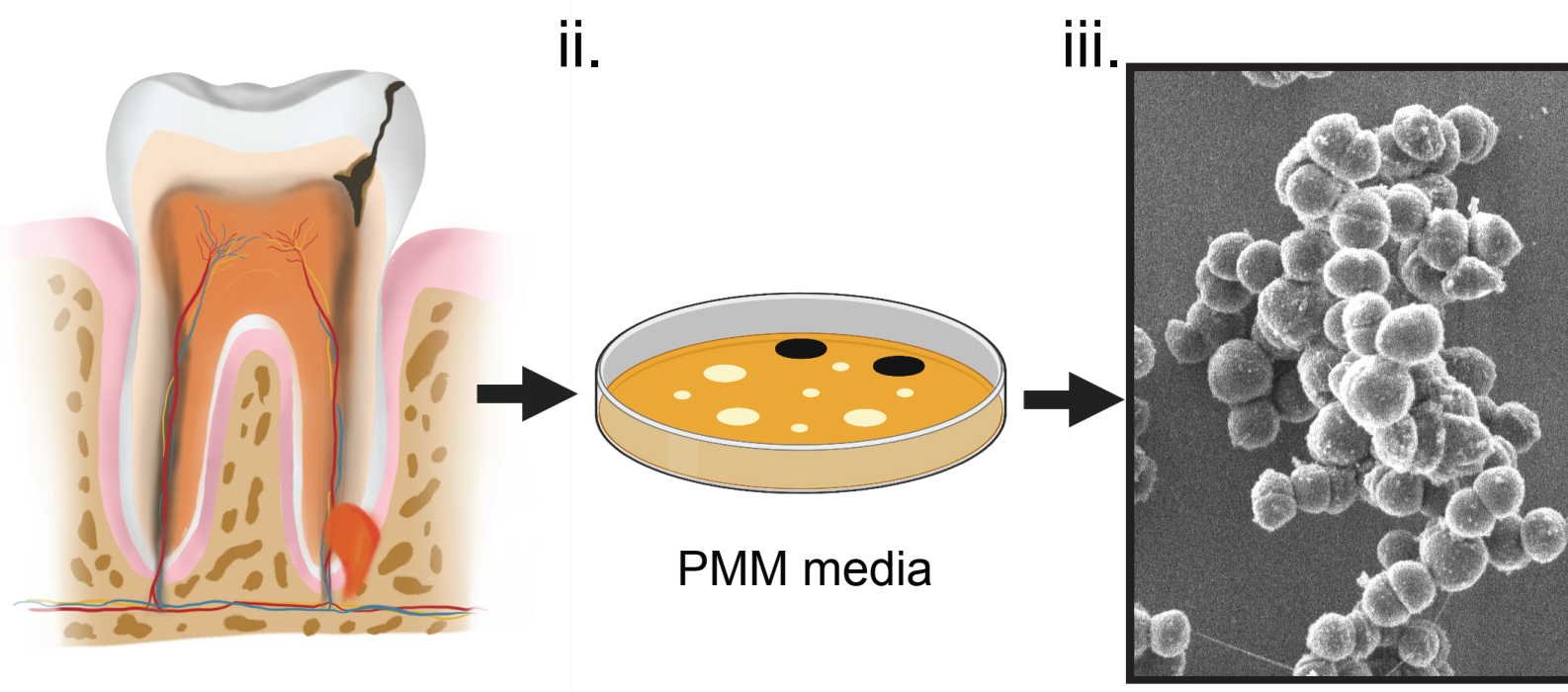

iv.

P. micra SEM

PMM media

P. TICra SEM

B i.

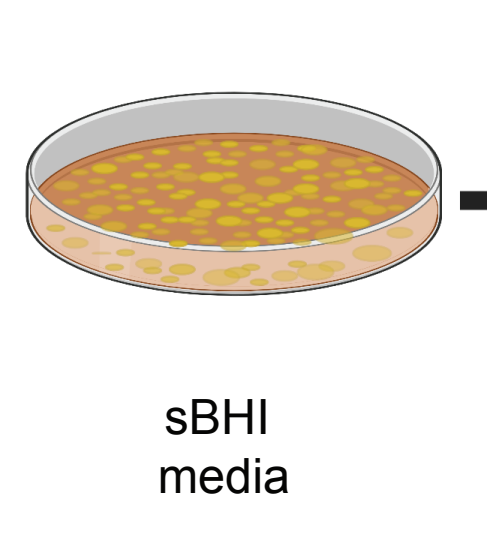

ii.

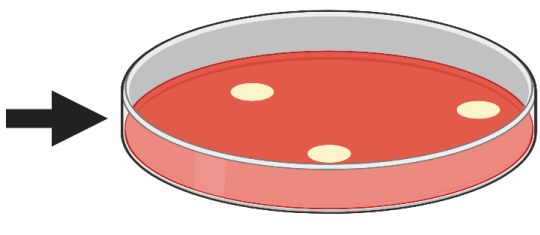

$$
\begin{gathered}
\mathrm{sBHI}+\text { rifampicin } \\
\text { media }
\end{gathered}
$$

iii.

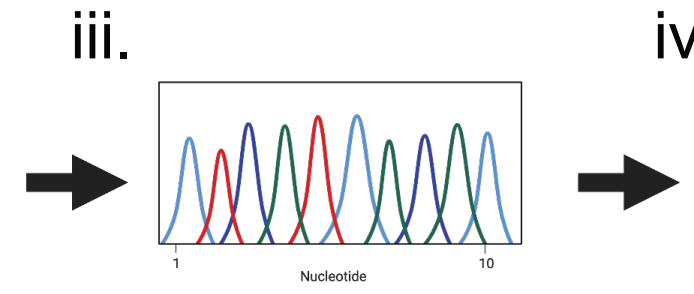

$r p o B$ sequencing

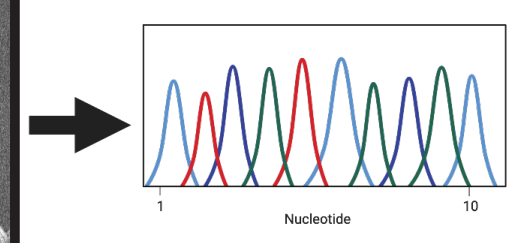

16S rRNA sequencing

iv.

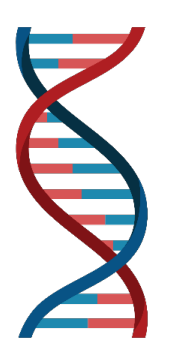

Transforming DNA

C

481

wt rif $^{S}$ RpoB RPLVASLKEFFGSSQLSQFMDQNNPLAELTHKRRLSALGPG rif $^{R}$ RpoB RPLVASLKEFFGSSQLSQFMVQNNPLAELTHKRRLSALGPG 


\section{Figure 3}

A
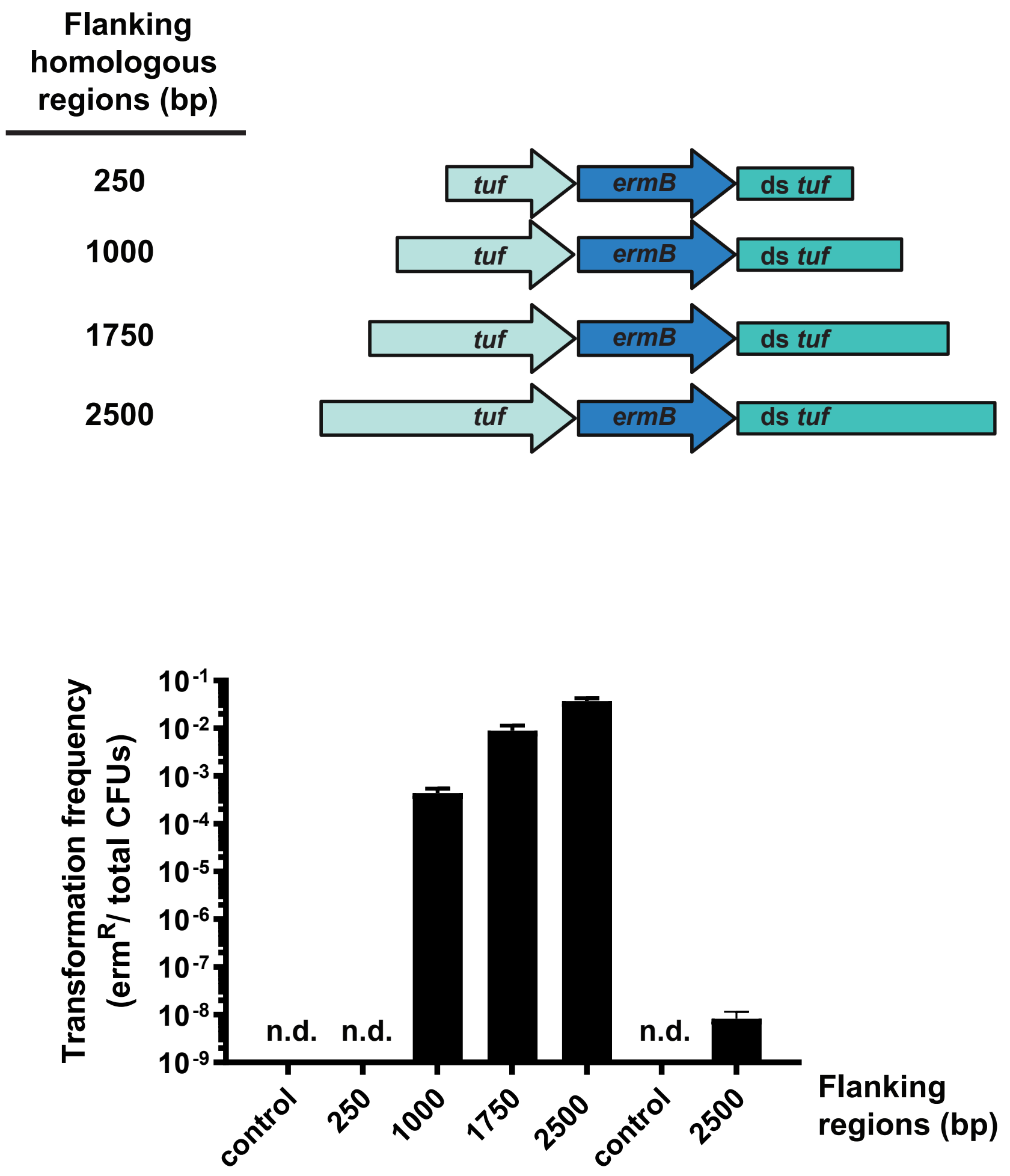

Flanking regions (bp) 
Figure 4

A

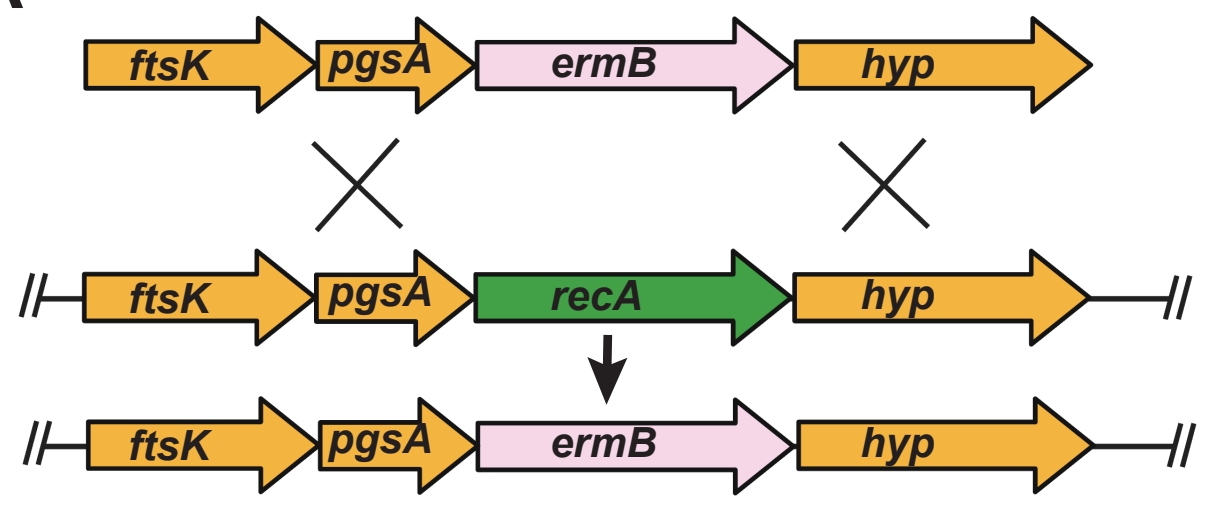

C

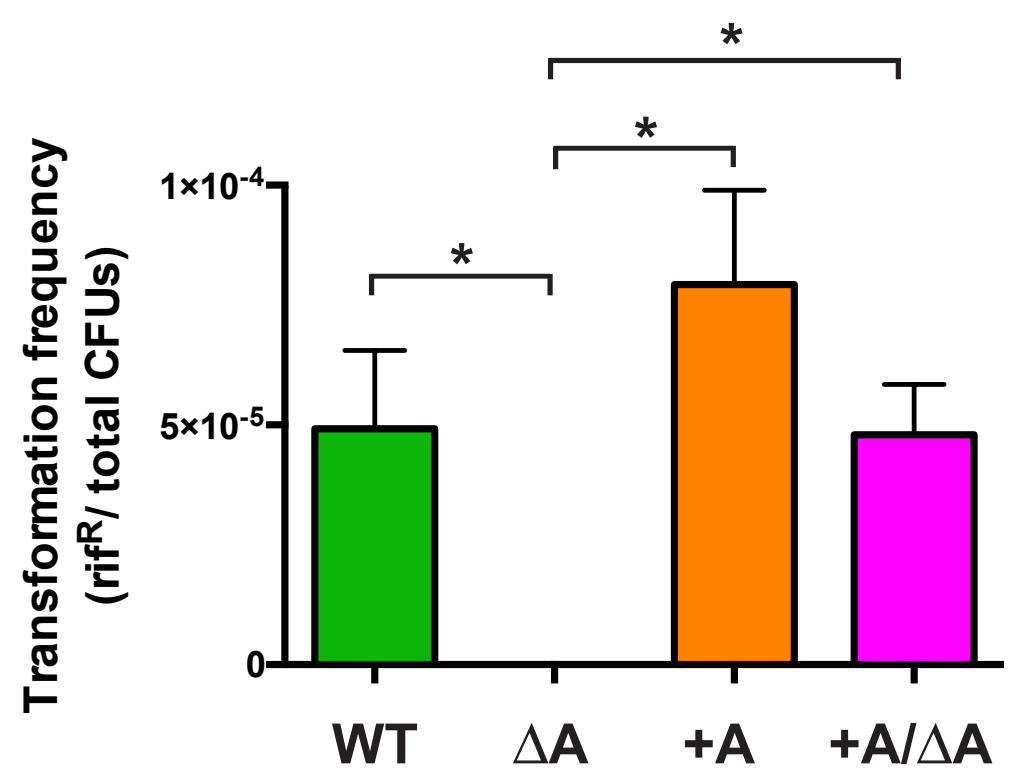

B

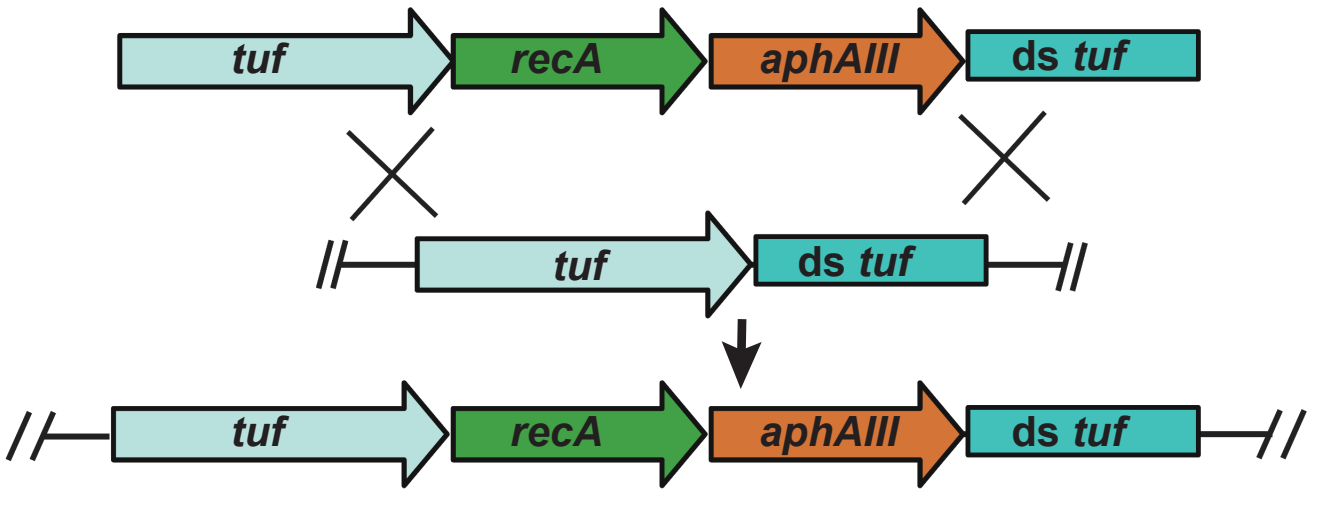

D

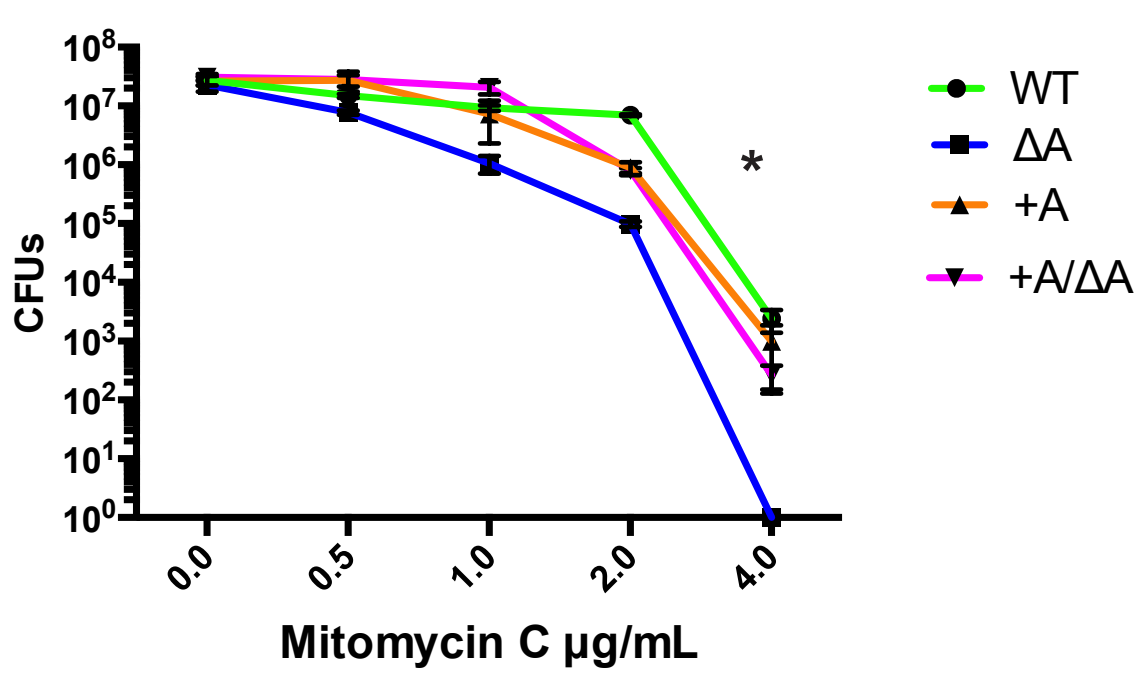




\section{Figure 5}

A

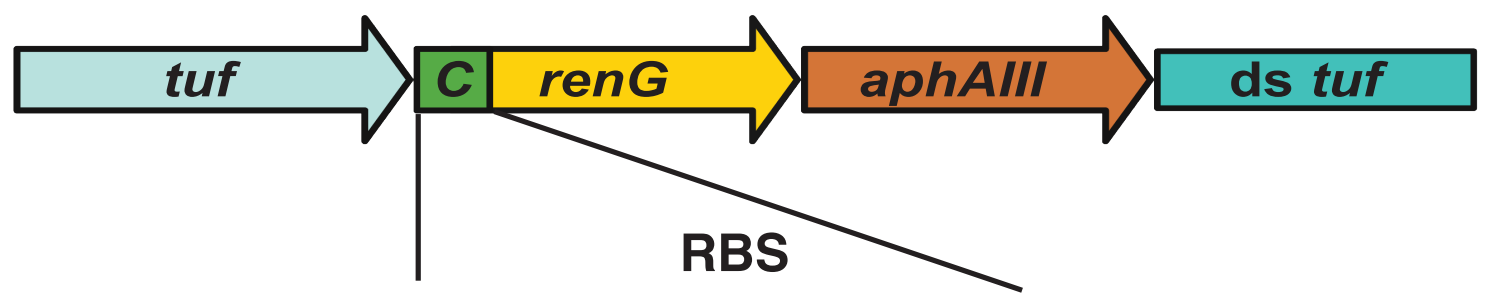

EF-Tu...TAAAATTTTAGGAGGATAAAATG...renG

B

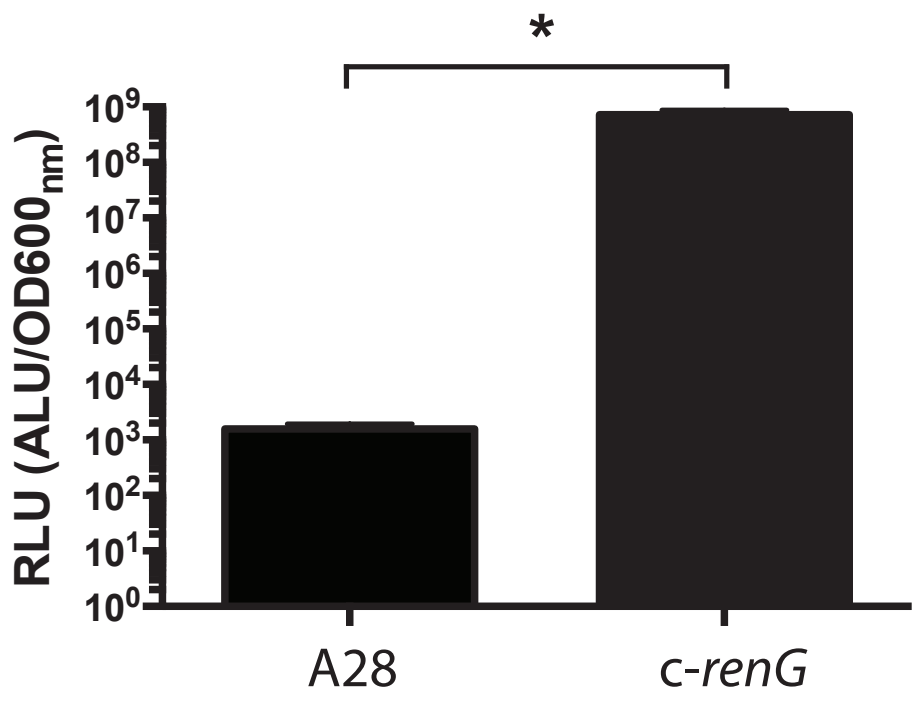

C

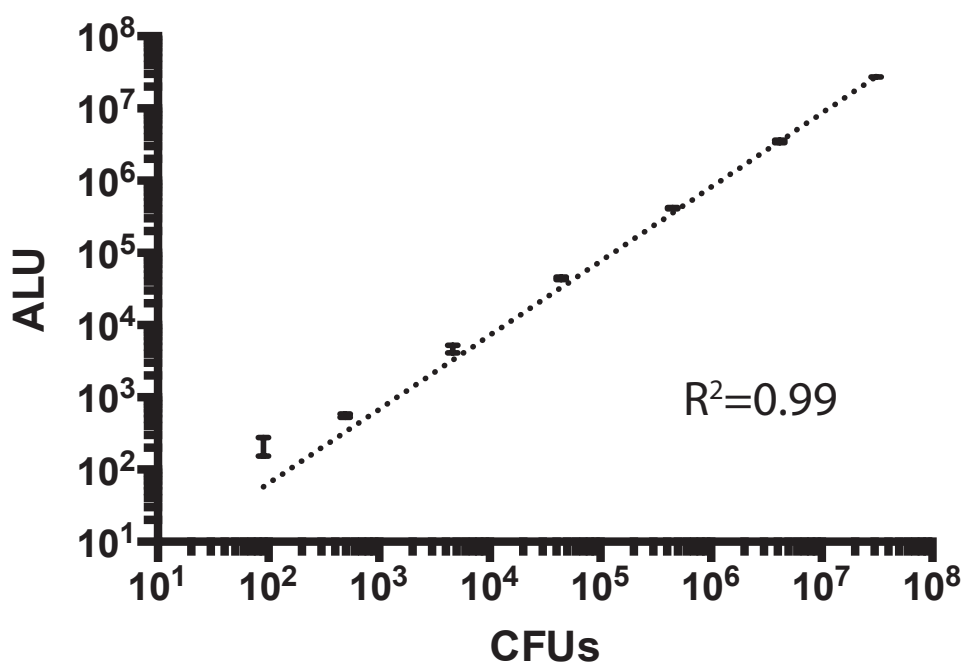




\section{Figure 6}

A

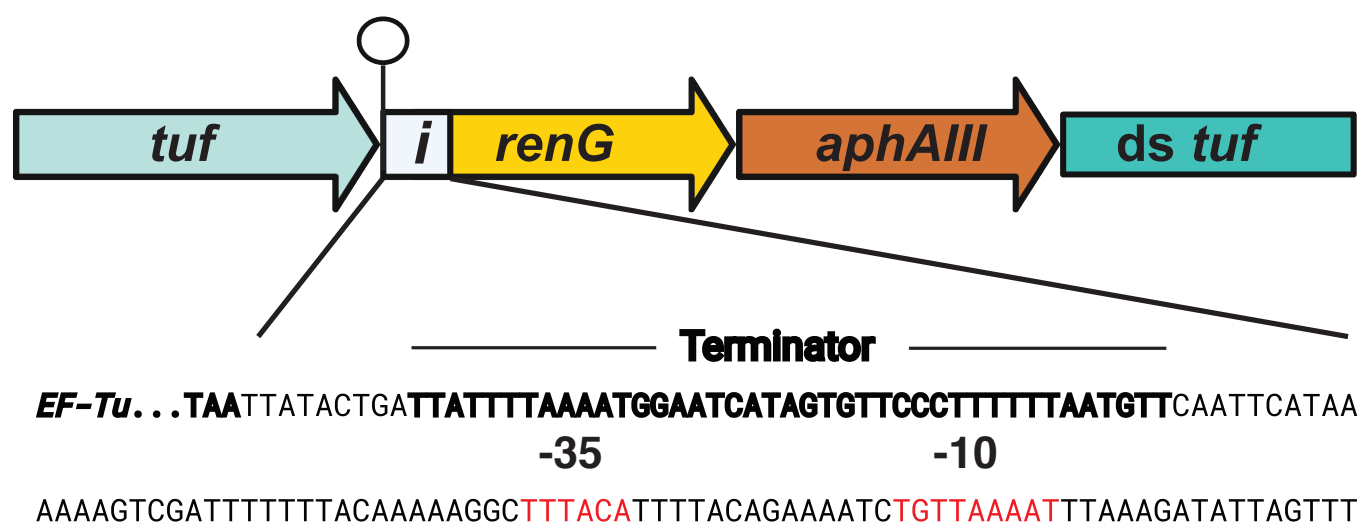

ATTGGATAAACAAACTAACTCAATTAAGATAGTTGATGGATAAACTTGTTCACTTAAATCGGTGATACCAG

RBS

CATCGTCTTGATGCCCTTGGCAGCACCCTGCTAAGGAGGTAACAACAAGATG . . . reng

Theo+ TTTTTT

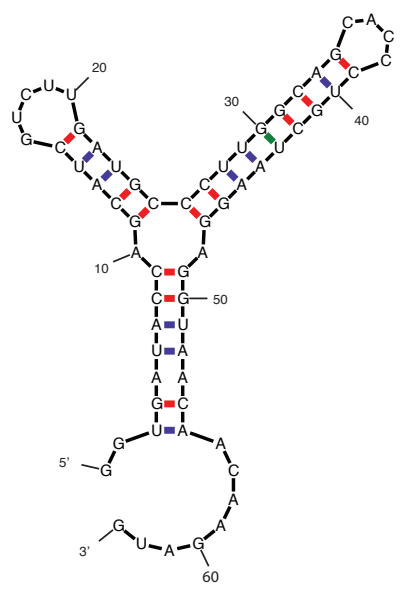

Theophylline Riboswitch

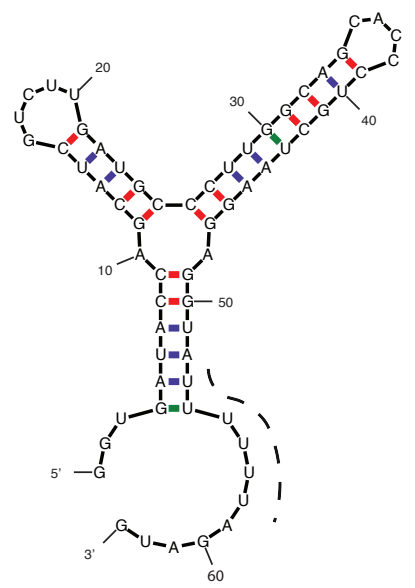

Theo+ Riboswitch

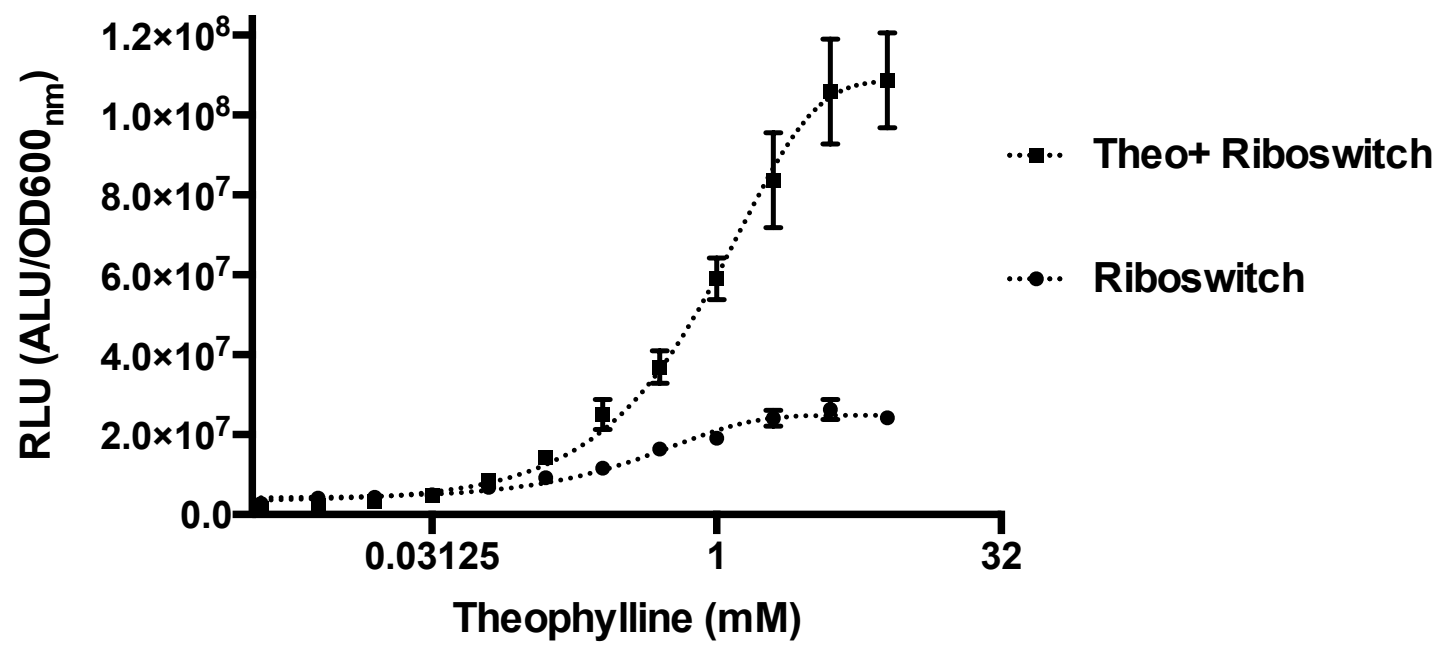




\section{Figure 7}

A

B

Transposase
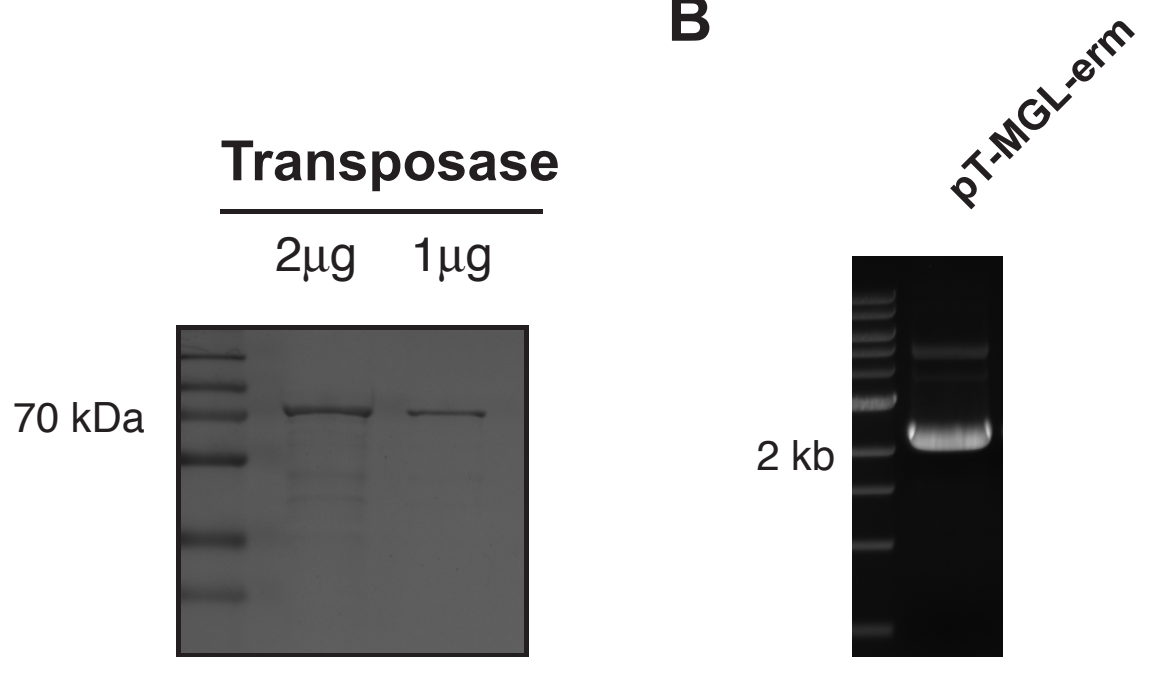

C

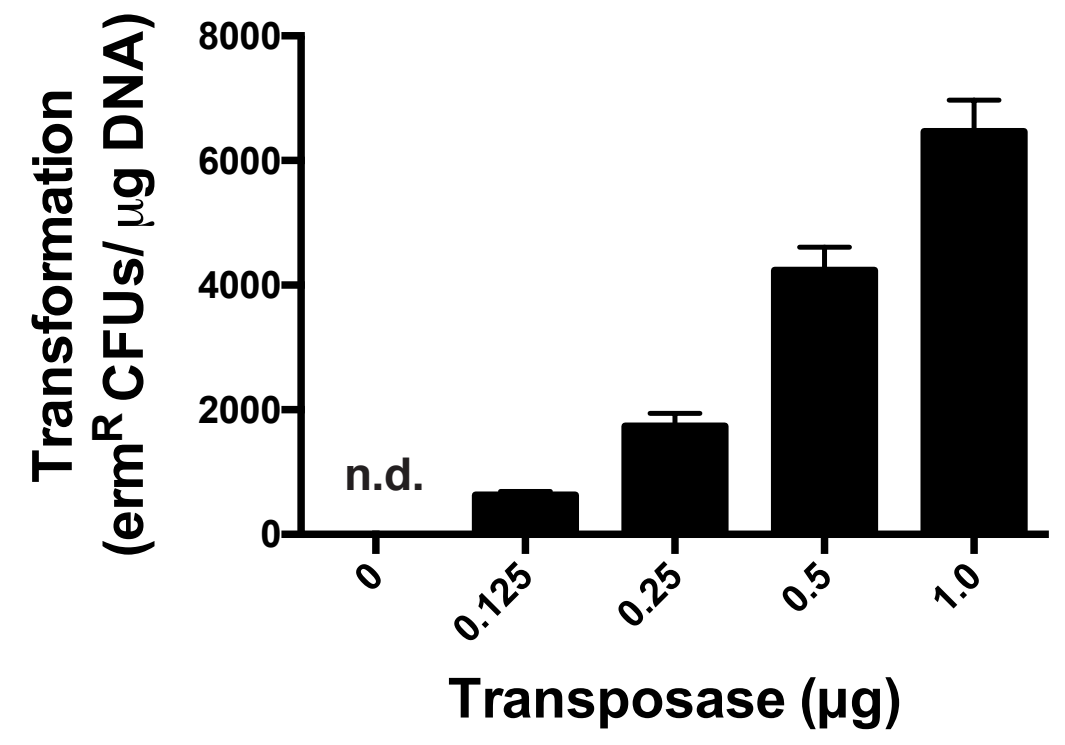

D

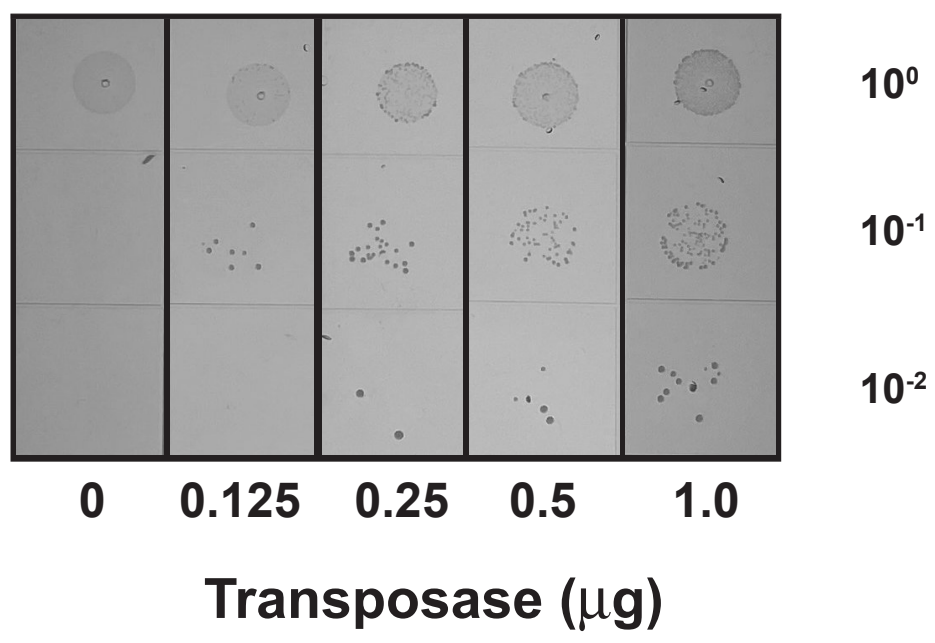




\section{Table 1}

\begin{tabular}{|c|c|c|c|}
\hline $\begin{array}{l}\text { Parvimonas micra } \\
\text { Isolate }\end{array}$ & $\begin{array}{l}\text { ATCC } \\
33270 \\
\text { gDNA } \\
\left(\text { rif }^{\mathrm{R}}\right) \\
\end{array}$ & 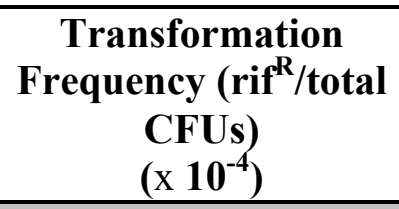 & $\begin{array}{c}\text { Fold-increase } \\
\text { (over } \\
\text { background) }\end{array}$ \\
\hline $\mathrm{A} 28$ & + & $1.73 \pm 0.34 *$ & 752 \\
\hline A28 & - & $0.0023 \pm 0.0005$ & \\
\hline A42 & + & $0.33 \pm 0.12 *$ & 236 \\
\hline A42 & - & $0.0014 \pm 0.0002$ & \\
\hline A11 & + & $0.26 \pm 0.06 *$ & 263 \\
\hline A11 & - & $0.00099 \pm 0.00014$ & \\
\hline ATCC 33270 & + & $0.075 \pm 0.0029 *$ & 36 \\
\hline ATCC 33270 & - & $0.0021 \pm 0.0022$ & \\
\hline A3 & + & $0.013 \pm 0.00013 *$ & 8 \\
\hline A3 & - & $0.0016 \pm 0.00043$ & \\
\hline A1 & + & $0.0040 \pm 0.0012^{\text {n.s. }}$ & 1 \\
\hline A1 & - & $0.0035 \pm 0.0014$ & \\
\hline
\end{tabular}

Transformation frequency is expressed as the number of rifampcin resistant bacteria/ total CFUs. Values are averaged from triplicate determinations \pm SD. Statistical analysis was performed using a two-tailed Student's $t$-test.

“*” indicates significance $(p<0.05)$ relative to wt gDNA control. "n.s." indicates no significance $(\mathrm{p}>0.60)$. 